\title{
Hierarchical frequency clusters in adaptive networks of phase oscillators
}

Cite as: Chaos 29, 103134 (2019); https://doi.org/10.1063/1.5097835

Submitted: 28 March 2019 . Accepted: 03 October 2019 . Published Online: 23 October 2019

Rico Berner (D), Jan Fialkowski (D), Dmitry Kasatkin (D), Vladimir Nekorkin (D), Serhiy Yanchuk (D), and Eckehard Schöll (D)

\section{COLLECTIONS}

Paper published as part of the special topic on Symmetry and Optimization in the Synchronization and Collective Behavior of Complex Systems

Note: This paper is part of the Focus Issue on Symmetry and Optimization in the Synchronization and Collective Behavior of Complex Systems.
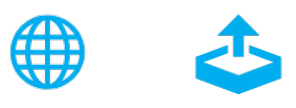

\section{ARTICLES YOU MAY BE INTERESTED IN}

Multistability and basin stability in coupled pendulum clocks

Chaos: An Interdisciplinary Journal of Nonlinear Science 29, 103140 (2019); https://

doi.org/10.1063/1.5118726

Solitary states and partial synchrony in oscillatory ensembles with attractive and repulsive interactions

Chaos: An Interdisciplinary Journal of Nonlinear Science 29, 093124 (2019); https:// doi.org/10.1063/1.5118843

Asymmetrical voltage response in resonant neurons shaped by nonlinearities

Chaos: An Interdisciplinary Journal of Nonlinear Science 29, 103135 (2019); https:// doi.org/10.1063/1.5110033

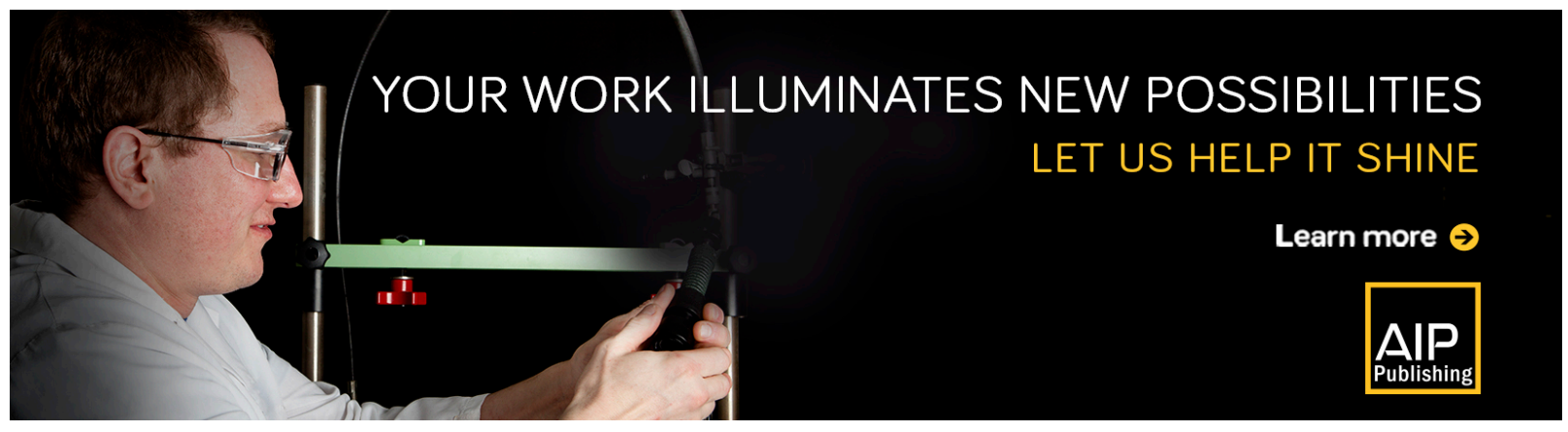




\title{
Hierarchical frequency clusters in adaptive networks of phase oscillators
}

\author{
Cite as: Chaos 29, 103134 (2019); doi: 10.1063/1.5097835 \\ Submitted: 28 March 2019 . Accepted: 3 October 2019. \\ Published Online: 23 October 2019

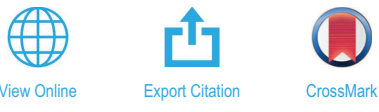

Rico Berner, ${ }^{1,2, a)}$ (D) Jan Fialkowski, 'D Dmitry Kasatkin, ${ }^{3}$ (D) Vladimir Nekorkin, ${ }^{3}$ (D) Serhiy Yanchuk, ${ }^{2}$ and Eckehard Schöll' (D)

\begin{abstract}
AFFILIATIONS
${ }^{1}$ Institute of Theoretical Physics, Technische Universität Berlin, Hardenbergstr. 36, D-10623 Berlin, Germany

${ }^{2}$ Institute of Mathematics, Technische Universität Berlin, Strasse des 17. Juni 136, D-10623 Berlin, Germany

${ }^{3}$ Institute of Applied Physics of RAS, 46 Ul'yanov Street, 603950 Nizhny Novgorod, Russia
\end{abstract}

\begin{abstract}
Note: This paper is part of the Focus Issue on Symmetry and Optimization in the Synchronization and Collective Behavior of
\end{abstract} Complex Systems.

a)rico.berner@physik.tu-berlin.de

\begin{abstract}
Adaptive dynamical networks appear in various real-word systems. One of the simplest phenomenological models for investigating basic properties of adaptive networks is the system of coupled phase oscillators with adaptive couplings. In this paper, we investigate the dynamics of this system. We extend recent results on the appearance of hierarchical frequency multiclusters by investigating the effect of the time scale separation. We show that the slow adaptation in comparison with the fast phase dynamics is necessary for the emergence of the multiclusters and their stability. Additionally, we study the role of double antipodal clusters, which appear to be unstable for all considered parameter values. We show that such states can be observed for a relatively long time, i.e., they are metastable. A geometrical explanation for such an effect is based on the emergence of a heteroclinic orbit.
\end{abstract}

Published under license by AIP Publishing. https://doi.org/10.1063/1.5097835

\begin{abstract}
Adaptive networks are characterized by the property that their connectivity can change in time, depending on the state of the network. A prominent example of adaptive networks are neuronal networks with plasticity, i.e., an adaptation of the synaptic coupling. Such an adaptation is believed to be related to learning and memory mechanisms. In other real-world systems, the adaptivity plays an important role as well. ${ }^{1}$ This paper investigates a phenomenological model of adaptively coupled phase oscillators. The considered model is a natural extension of the Kuramoto system to the case with dynamical couplings. In particular, we review and provide new details on the self-organized emergence of multiple frequency clusters.
\end{abstract}

\section{INTRODUCTION}

One of the main motivations for studying adaptive dynamical networks comes from the field of neuroscience where the weights of the synaptic coupling can adapt depending on the activity of the neurons that are involved in the coupling. ${ }^{2-4}$ For instance, the coupling weights can change in response to the relative timings of neuronal spiking. ${ }^{5-8}$ Adaptive networks appear also in chemical, ${ }^{9}$ biological, or social systems.

This paper is devoted to a simple phenomenological model of adaptively coupled phase oscillators. The model has been extensively studied recently, ${ }^{10-20}$ and it exhibits diverse complex dynamical behavior. In particular, stable multifrequency clusters emerge in this system, when the oscillators split into groups of strongly coupled oscillators with the same average frequency. Such a phenomenon does not occur in the classical Kuramoto or Kuramoto-Sakaguchi system. The clusters are shown to possess a hierarchical structure, i.e., their sizes are significantly different. ${ }^{20}$ Such a structure leads to significantly different frequencies of the clusters and, as a result, to their uncoupling. This phenomenon is reported for an adaptive network of Morris-Lecar bursting neurons with the spike-timing-dependent plasticity rule. ${ }^{21}$ In addition, the role of hierarchy and modularity in brain networks has been discussed recently. ${ }^{22-26}$ Both features, therefore, seem to play a key role in real-world neural networks. Along these lines, we study a dynamical model to analyze the self-organized formation of such network structures. 
In this paper, we first provide a nontechnical overview of known results on multifrequency clusters from Refs. 10 and 20. Apart from that, we investigate the role of the time scale separation. Particularly, we find that the slow adaptation mechanism in comparison with the fast dynamics of the oscillators is an important necessary ingredient for the emergence of stable multiclusters. Discussing the stability, we point out that the stability analysis for the limiting case without adaptation does not provide correct stability results for arbitrarily small adaptation. In addition, the stability for one-clusters is described depending on the time scale separation. With these results, we quantitatively relate the stability of one- with multifrequency cluster states which goes beyond the qualitative analysis given in Ref. 20. Providing a novel result for the stability of two-clusters, the instability of evenly sized clusters is shown. Finally, we discuss the role of a special type of cluster states, called "double antipodal" clusters. We show that these states are unstable for all parameter values but can appear as saddles connecting synchronous and splay states in phase space. As a result, such states can be observed as a "metastable" transition between the phase-synchronous and the nonphase-synchronous state. Moreover, the double antipodal states are shown to play an important role in the global dynamics of the adaptive system.

The structure of the paper is as follows. Section II presents the model; Secs. III and IV provide a nontechnical overview of Ref. 20 on the multifrequency cluster states and their classification. The new results are included in Secs. V-VIII. In Sec. IV, we further describe one-clusters, i.e., single blocks of which the multiclusters are composed. Section V discusses the results of Ref. 20 on the stability of one- and multifrequency clusters from a different viewpoint focusing on the influence of the time scale separation. We provide novel rigorous results on the stability for the whole classes of antipodal, double antipodal, and 4-phase cluster states. The stability regions for antipodal and splay-type one-clusters are explicitly described for any parameter range of the time scale separation. In Sec. VI, the role of double antipodal states for the global dynamics of the system is discussed. The construction of multicluster states from one-clusters is demonstrated in Sec. VII. For the existence of multiclusters,. a new upper bound for the time separation is derived. In Sec. VIII, we connect the stability properties of one- and multiclusters. As conjectured in Ref. 20, the instability of evenly sized two-clusters of splay type is proved. For the sake of readability, the proofs for any of the statements in this section are provided in the Appendix. We end with the conclusion.

\section{MODEL}

In this article, we consider a network of $N$ adaptively coupled phase oscillators,

$$
\begin{aligned}
& \frac{d \phi_{i}}{d t}=\omega-\frac{1}{N} \sum_{j=1}^{N} \kappa_{i j} \sin \left(\phi_{i}-\phi_{j}+\alpha\right), \\
& \frac{d \kappa_{i j}}{d t}=-\epsilon\left(\sin \left(\phi_{i}-\phi_{j}+\beta\right)+\kappa_{i j}\right),
\end{aligned}
$$

where $\phi_{i} \in[0,2 \pi)$ is the phase of the $i$ th oscillator $(i=1, \ldots, N)$ and $\omega$ is the natural frequency. The oscillators interact according to the coupling structure represented by the coupling weights $\kappa_{i j}(i, j=1, \ldots, N)$ as dynamical variables. The parameter $\alpha$ can be (a)

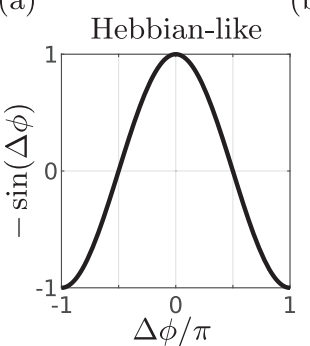

(b)

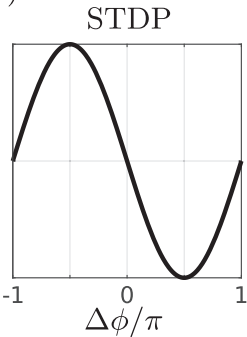

(c)

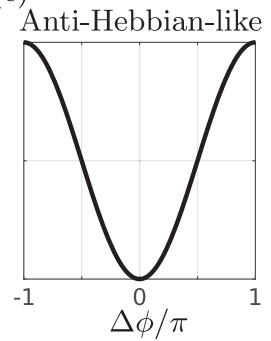

FIG. 1. The plasticity function $-\sin (\Delta \phi+\beta)$ and corresponding plasticity rules are presented. (a) $\beta=-\frac{\pi}{2}$ (Hebbian), (b) $\beta=0$ (spike timing-dependent plasticity, STDP), (c) $\beta=\frac{\pi}{2}$ (anti-Hebbian).

considered as a phase lag of the interaction. ${ }^{27}$ This paradigmatic model of an adaptive network equations (1) and (2) has attracted a lot of attention recently. ${ }^{10-20}$ It provides a generalization of the Kuramoto-Sakaguchi model with fixed $\kappa_{i j}{ }^{28-32}$

The coupling topology of the network at time $t$ is characterized by the coupling weights $\kappa_{i j}(t)$. With a small parameter $0<\epsilon$ $\ll 1$, the dynamical equation (2) describes the adaptation of the network topology depending on the dynamics of the network nodes. In the neuroscience context, such an adaptation can also be called plasticity. ${ }^{12}$ The chosen adaptation function has the form $-\sin \left(\phi_{i}-\right.$ $\phi_{j}+\beta$ ) with control parameter $\beta$. With this, different plasticity rules can be modeled, see Fig. 1. For instance, for $\beta=-\pi / 2$, a Hebbianlike rule is obtained where the coupling $\kappa_{i j}$ is increasing between any two phase oscillators with close phases, i.e., $\phi_{i}-\phi_{j}$ close to zero ${ }^{33-36}$ (fire together-wire together). If $\beta=0$, the link $\kappa_{i j}$ is strengthened if the $j$ th oscillator precedes the $i$ th. Such a relationship promotes a causal structure in the oscillatory system. In neuroscience, these adaptation rules are typical for spike timing-dependent plasticity. $7,8,37,38$

Let us mention important properties of the model (1) and (2). The parameter $\epsilon \ll 1$ separates the time scales of the slowly adapting coupling weights from the fast moving phase oscillators. Further, the coupling weights are confined to the interval $-1 \leq \kappa_{i j} \leq 1$ due to the fact that $d \kappa_{i j} / d t \leq 0$ for $\kappa_{i j}=1$ and $d \kappa_{i j} / d t \geq 0$ for $\kappa_{i j}=-1$, see Ref. 10. Due to the invariance of system (1) and (2) with respect to the $\operatorname{shift} \phi_{i} \mapsto \phi_{i}+\psi$ for all $i=1, \ldots, N$ and $\psi \in[0,2 \pi)$, the frequency $\omega$ can be set to zero in the corotating coordinate frame $\phi \mapsto \phi+\omega t$. Finally, we mention the symmetries of the system (1) and (2) with respect to the parameters $\alpha$ and $\beta$,

$$
\begin{aligned}
& \left(\alpha, \beta, \phi_{i}, \kappa_{i j}\right) \mapsto\left(-\alpha, \pi-\beta,-\phi_{i}, \kappa_{i j}\right), \\
& \left(\alpha, \beta, \phi_{i}, \kappa_{i j}\right) \mapsto\left(\alpha+\pi, \beta+\pi, \phi_{i},-\kappa_{i j}\right) .
\end{aligned}
$$

These symmetries allow for a restriction of the analysis to the parameter region $\alpha \in[0, \pi / 2)$ and $\beta \in[-\pi, \pi)$.

The Kuramoto order parameter $R_{1}$ measures the synchrony of phase oscillators $\boldsymbol{\phi}=\left(\phi_{1}, \ldots, \phi_{N}\right)^{T}$. Correspondingly, the $l$ th moment order parameter $R_{l}$ is given by

$$
R_{l}(\boldsymbol{\phi}):=\left|\frac{1}{N} \sum_{j=1}^{N} e^{\mathrm{i} l \phi_{j}}\right|,
$$


where $l \in \mathbb{N}$. In the following section, we will use this quantity in order to characterize several dynamical states.

\section{HIERARCHICAL FREQUENCY CLUSTERS}

System (1) and (2) has been studied numerically in Refs. 1014. In particular, it is shown that starting from uniformly distributed random initial condition $\left(\phi_{i} \in[0,2 \pi), \kappa_{i j} \in[-1,1]\right.$ for all $i, j \in$ $\{1, \ldots, N\})$, the system can reach different multifrequency cluster states with hierarchical structure. An individual cluster in the multicluster state consists of frequency synchronized groups of phase oscillators. In the following, we discuss the structural form of these clusters. Subsequently, frequency multicluster states are called multicluster states or multiclusters for simplicity.

Figure 2 shows a hierarchical multicluster state. The solution was obtained by integrating the system (1) and (2) numerically starting from uniformly distributed random initial conditions. The selfcouplings $\kappa_{i i}$ are set to zero in numerical simulations, since they

(a)

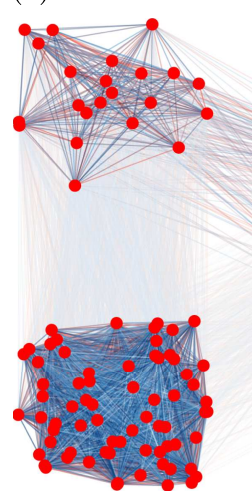

(b)

\section{Coupling network}

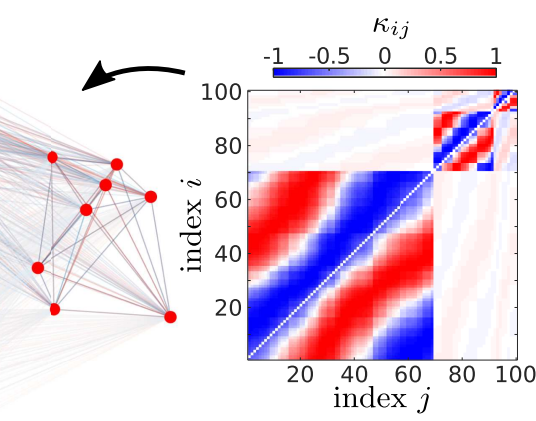

Oscillator phases

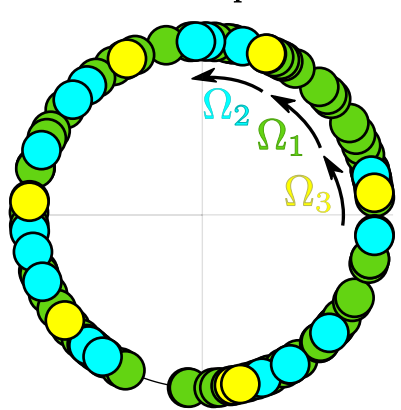

FIG. 2. Three-frequency cluster of splay type at $t=10000$. (a) Coupling weights represented as a graph (left) and as a coupling matrix (right). In the graph representation, the dynamical vertices are represented by red nodes and the edges are colored with respect to the coupling weight. Red and blue refer to positive and negative coupling weights, respectively. Light and dark colors refer to weak and strong coupling weights, respectively. (b) Distribution of the phases $\phi_{i}$ for each of the three clusters. Each node represents one oscillator and is colored with respect to the cluster to which it belongs. Parameter values: $\epsilon=0.01, \alpha=0.3 \pi$, $\beta=0.23 \pi, \omega=0$, and $N=100$. do not influence the relative dynamics of the system. ${ }^{39}$ We reorder the oscillators (after sufficiently long transient time) by first sorting the oscillators with respect to their average frequencies. After that, the oscillators with the same frequency are sorted by their phases. Figure 2(a) displays the coupling matrix (right) of the multicluster state and a representation of the coupling structure as a network graph (left). The coupling matrix demonstrates a clear splitting into three groups. This splitting is also visible in the graph representation of the coupling network. The coupling weights between oscillators of the same group vary in a larger range than between those of different groups which are generally smaller in magnitude. The splitting into three groups is manifested in the behavior of the phase oscillators, as well. We find that the oscillators of the same group possess the same constant frequency with possible phase lags, Fig. 2(b). We call the groups of oscillators (frequency) clusters and the corresponding dynamical states multi(frequency)cluster states.

In a multicluster, the coupling matrix $\kappa$ can be divided into different blocks; $\kappa_{i j, \mu \nu}$ will refer to the coupling weight between the $i$ th oscillator of the $\mu$ th cluster to the $j$ th oscillator of the $v$ th cluster. Analogously, $\phi_{i, \mu}$ denotes the $i$ th phase oscillator in the $\mu$ th cluster. In general, the temporal behavior for each oscillator in an M-cluster state takes the form

$$
\phi_{i, \mu}(t)=\Omega_{\mu} t+a_{i, \mu}+s_{i, \mu}(t), \quad \begin{aligned}
& \mu=1, \ldots, M \\
& i=1, \ldots, N_{\mu}
\end{aligned}
$$

where $M$ is the number of clusters, $N_{\mu}$ is the number of oscillators in the $\mu$ th cluster, $a_{i, \mu} \in[0,2 \pi)$ are phase lags, and $\Omega_{\mu} \in \mathbb{R}$ is the collective frequency of the oscillators in the $\mu$ th cluster. The functions $s_{i, \mu}$ are bounded.

The numerical analysis of system (1) and (2) shows the appearance of different multicluster states depending on particular choices of the phase lag parameters $\alpha$ and $\beta$ as well as on initial conditions. Starting from random initial conditions, the system can end up in several states such as multiclusters and chimeralike states. ${ }^{10}$ Figure 3 shows examples for the three types of multicluster states which appear dynamically in (1) and (2).

\section{A. Splay type multiclusters}

The first type is called splay type multicluster state, see Fig. 3(a). The separation into three clusters is clearly visible in the coupling matrix, as well as a hierarchical structure in the cluster sizes. Regarding the distribution of the phases, we note that the oscillators from each group are almost homogeneously dispersed on the circle. In fact, the phases from each cluster fulfill the condition $R_{2}\left(\boldsymbol{\phi}_{\mu}\right)=0$ $(\mu=1,2,3)$. Note that splay states as they are defined in several other works ${ }^{40-42}$ share the property $R_{1}(\boldsymbol{\phi})=0$. This property can be seen as a measure of incoherence for the oscillator phases, as well. In fact, it was shown that splay states are part of a whole family of solutions ${ }^{43,44}$ given by exactly $R_{1}(\phi)=0$. Further, $R_{2}(\phi)=R_{1}(2 \phi)$ relates the two measures of incoherence. These facts motivate the definition of those clusters with $R_{2}(\boldsymbol{\phi})=0$ as "splay-type clusters."

The temporal behavior for all phase oscillators in the splay multicluster state is characterized by a constant frequency which differs for the different clusters, i.e., according to (4), $\phi_{i, \mu}(t)=$ $\Omega_{\mu} t+a_{i, \mu}$ with $R_{2}\left(\boldsymbol{a}_{\mu}\right)=0$ for all $\mu=1,2,3$ and $i=1, \ldots, N_{\mu}$. 

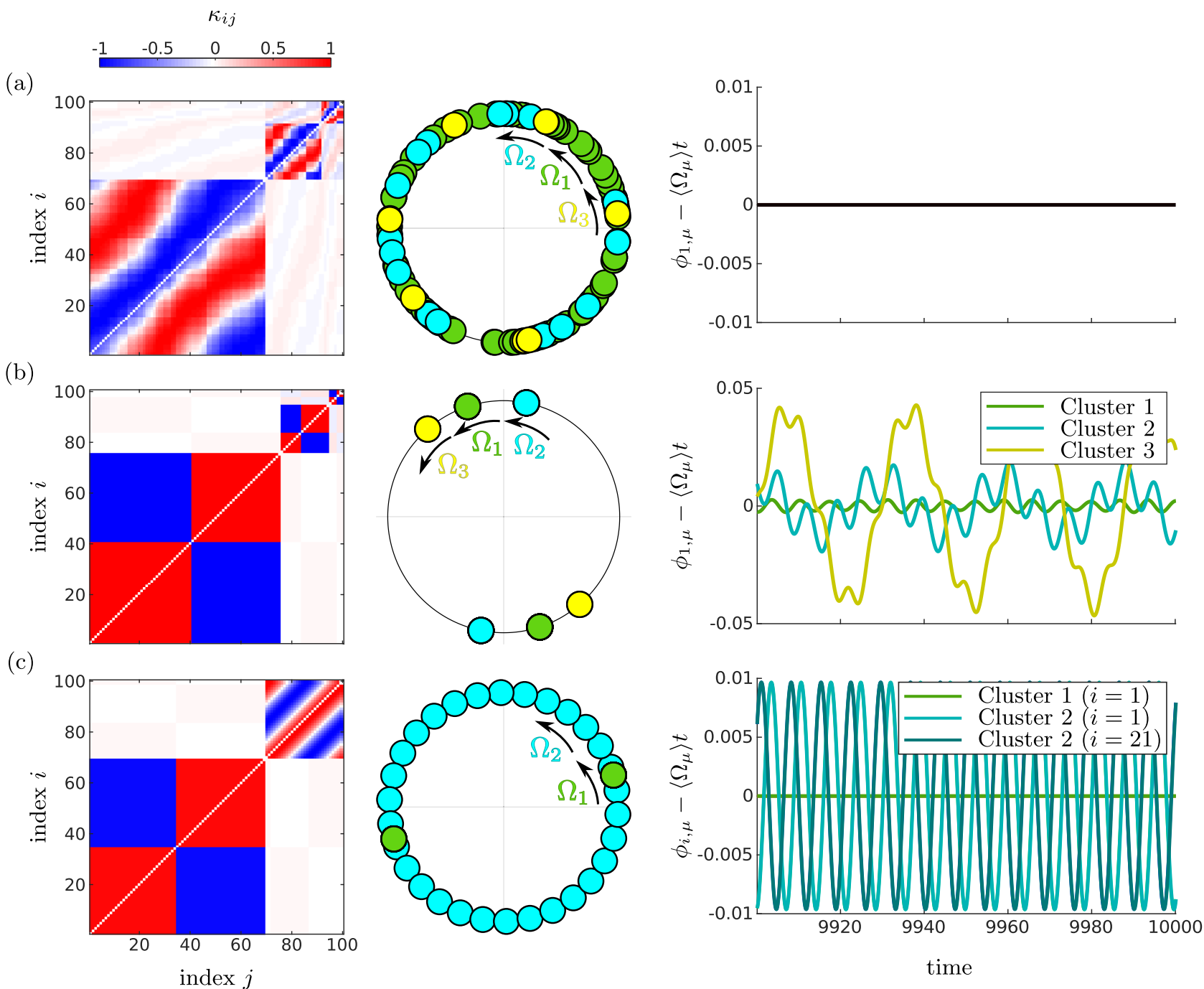

FIG. 3. Three different types of multicluster states at $t=10000$ with $N=100$ and $\epsilon=0.01$. For all types, the coupling matrix (left), distribution of the phases (middle), and time series of representative phase oscillators from each cluster (right) are presented. In the plot of the phase distribution, each node represents one oscillator and is colored with respect to the cluster to which it belongs. The time series are shown after subtracting the average linear growth $\phi_{i, \mu}(t)-\left\langle\Omega_{\mu}\right\rangle t$. The coloring of the time series (shaded for visibility) of a representative phase oscillator from one-cluster is in accordance with the pictures in the middle panel. (a) Splay type 3 -cluster for $\alpha=0.3 \pi, \beta=0.23 \pi$; (b) antipodal type 3-cluster for $\alpha=0.3 \pi, \beta=-0.53 \pi$; (c) mixed type 2-cluster for $\alpha=0.3 \pi, \beta=-0.4 \pi$.

In addition, the hierarchical cluster sizes are reflected in the frequencies. Oscillators of a big cluster have a higher frequency than those of smaller clusters. The coupling weights between the phase oscillators are fixed or change periodically with time depending on whether the oscillators belong to the same or different clusters, respectively. Moreover, the amplitude of coupling weights between clusters depends on the frequency difference of the corresponding clusters. The higher the frequency difference, the smaller is the amplitude. The periodic behavior of the coupling weights between clusters is present in all types of multicluster states [Figs. 3(a)-3(c)].

\section{B. Antipodal type multiclusters}

Figure 3(b) shows another possible multicluster state. As in Fig. 3(a), the clusters are clearly visible and their oscillators show frequency synchronized temporal behavior. In addition, the time series for the oscillators show periodic modulations on top of the linear growth. This additional dynamics is the same for all oscillators of the same cluster, and hence they are still temporally synchronized. We have $\phi_{i, \mu}(t)=\Omega_{\mu} t+a_{i, \mu}+s_{\mu}(t)$. In analogy to the coupling weights between the clusters, the amplitudes of the bounded function $s_{\mu}(t)$ depend on the differences of the cluster frequencies. 
In contrast to the splay states, the phase distribution fulfills $R_{2}\left(\boldsymbol{a}_{\mu}\right)=1$ for all $\mu=1,2,3$, see Fig. 3(b), middle panel. Hence, all oscillators of a cluster have either the same phase $a_{\mu} \in[0,2 \pi)$ or the antipodal phase $a_{\mu}+\pi$ such that $2 a_{i, \mu}=2 a_{\mu}$ modulo $2 \pi$ for all $i=1, \ldots, N_{\mu}$. Therefore, the clusters represented in Fig. 3(b) are called "antipodal type multicluster." Note that with this formal definition of an antipodal state, in-phase clusters belong to the class of antipodal clusters.

\section{Mixed type multiclusters}

The third type of multicluster states combines the previous two types. The 2-cluster state shown in Fig. 3(c) consists of one splay cluster and one antipodal cluster. We call these states "mixed type multicluster states." As we have seen before, the interaction of a cluster with an antipodal cluster induces a modulation $s(t)$ additional to the linear growth of the oscillator's phase. In contrast, the interaction with a splay cluster does not introduce any modulation. Thus, the temporal dynamics of the oscillators in the antipodal cluster $(\mu=1)$ have $s_{i, 1}(t) \equiv 0$, while the oscillators in the splay cluster $(\mu=2)$ show additional bounded modulations $s_{i, 2}(t)$, see Fig. $3(\mathrm{c})$. For the oscillators of the splay cluster, we plot the time series of two representatives. We note the temporal shift in the dynamics of the two representatives of the splay cluster. The oscillators in the splay cluster are not completely temporally synchronized. More specifically, we have $\phi_{i, 1}(t)=\Omega_{1} t+a_{i, 1}$ with $R_{2}\left(\boldsymbol{a}_{1}\right)=1$ for $i=1, \ldots, N_{1}$ and $\phi_{i, 2}(t)=\Omega_{2} t+a_{i, 2}+s_{i, 2}(t)$ with $R_{2}\left(\boldsymbol{a}_{2}\right)=0$ for $i=1, \ldots, N_{2}$.

Despite the complexity of the three types of multicluster states, the structures can be broken down into simple blocks. In fact, onecluster states of splay and antipodal type serve as building blocks in order to create more complex multicluster structures. In the following section, we will analyze these blocks. The building of higher cluster structures will be discussed for 2-cluster states of splay type.

\section{ONE-CLUSTER STATES}

As we have seen in Sec. III, certain one-cluster states serve as building blocks for higher multicluster states. In this section, we review the basic properties of one-cluster states and conclude that their shape and existence is independent of the time-separation parameter, see Ref. 20 for more details.

Formally, a one-cluster state is one group of frequency synchronized phase oscillators given by

$$
\phi_{i}=\Omega t+a_{i},
$$

with $a_{i} \in[0,2 \pi)(i=1, \ldots, N)$ and constant coupling weights

$$
\kappa_{i j}=-\sin \left(a_{i}-a_{j}+\beta\right),
$$

where $i, j=1, \ldots, N$.

Figure 4 shows three possible types of one-cluster states for the system (1) and (2). It has been shown that these are the only existing types of one-cluster states. ${ }^{20}$ The first two shown in Figs. 4(a) and $4(\mathrm{~b})$ are the splay $\left[R_{2}(\mathbf{a})=0\right]$ and the antipodal $\left[R_{2}(\mathbf{a})=1\right]$ clusters which were already discussed in Sec. III. The third type in Fig. 4(c) consists of two groups of antipodal phase oscillators with a fixed phase lag $\psi$. We call this class of states "double antipodal." As it was mentioned in Sec. III B, the formal definition of an antipodal (a)
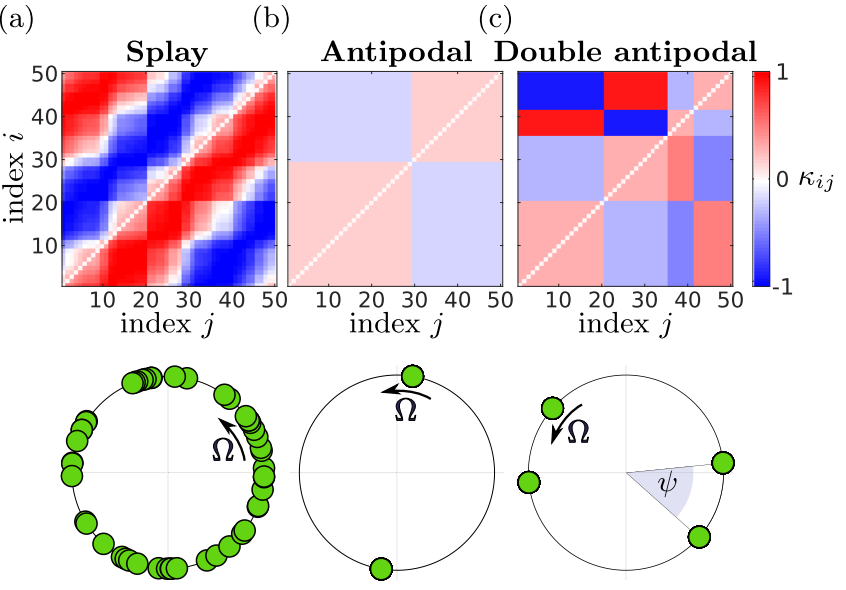

FIG. 4. All possible types of one-cluster states for system Eqs. (1) and (2). (a) Splay state; (b) antipodal state; (c) double antipodal state.

state includes full in-phase relation of the oscillators. Thus, in extension of the typical configuration shown in Fig. 4(c), there exist double antipodal states where only three or even only two different phases are occupied. The constant $\psi$ is the unique (modulo $2 \pi$ ) solution of the equation,

$$
\frac{1-q}{q} \sin (\psi-\alpha-\beta)=\sin (\psi+\alpha+\beta),
$$

where $q=Q / N$ and $Q$ is the number of phase shifts $a_{i}$ such that $a_{i} \in$ $\{0, \pi\}$. The corresponding frequencies for the three types of states are

$$
\Omega= \begin{cases}\frac{1}{2} \cos (\alpha-\beta) & \text { if } R_{2}(\mathbf{a})=0, \\ \sin \alpha \sin \beta & \text { if } R_{2}(\mathbf{a})=1, \\ \frac{1}{2}\left[\cos (\alpha-\beta)-R_{2}(\mathbf{a}) \cos \psi\right] & \text { if double antipodal. }\end{cases}
$$

Note that the condition $R_{2}(\mathbf{a})=0$ gives rise to an $N-2$ dimensional family of solutions. Well-known representatives of this family are rotating-wave states which have the following form $\mathbf{a}_{k}=(0,2 \pi k / N, \ldots, 2 \pi k(N-1) / N)^{T}$ for any wavenumber $k=1, \ldots, N-1$. The existence as well as the explicit form for any of the three types of one-cluster states do not depend on the time separation parameter $\epsilon$. As long as $\epsilon>0$, the building blocks appear to be solutions of the system (1) and (2).

\section{STABILITY OF ONE-CLUSTER STATES}

In Secs. III and IV, a large number of coexisting multicluster states were discussed. As multiclusters are constructed out of one-cluster states, studying the stability of the building blocks is of major importance. In contrast to the existence of one-cluster states, the stability of those states depends crucially on the time-separation parameter. We analyze this dependency below.

The diagram in Fig. 5 shows the regions of stability for antipodal and rotating-wave one-cluster states. The diagram is based on analytic results which have been recently found. In the Appendix, 


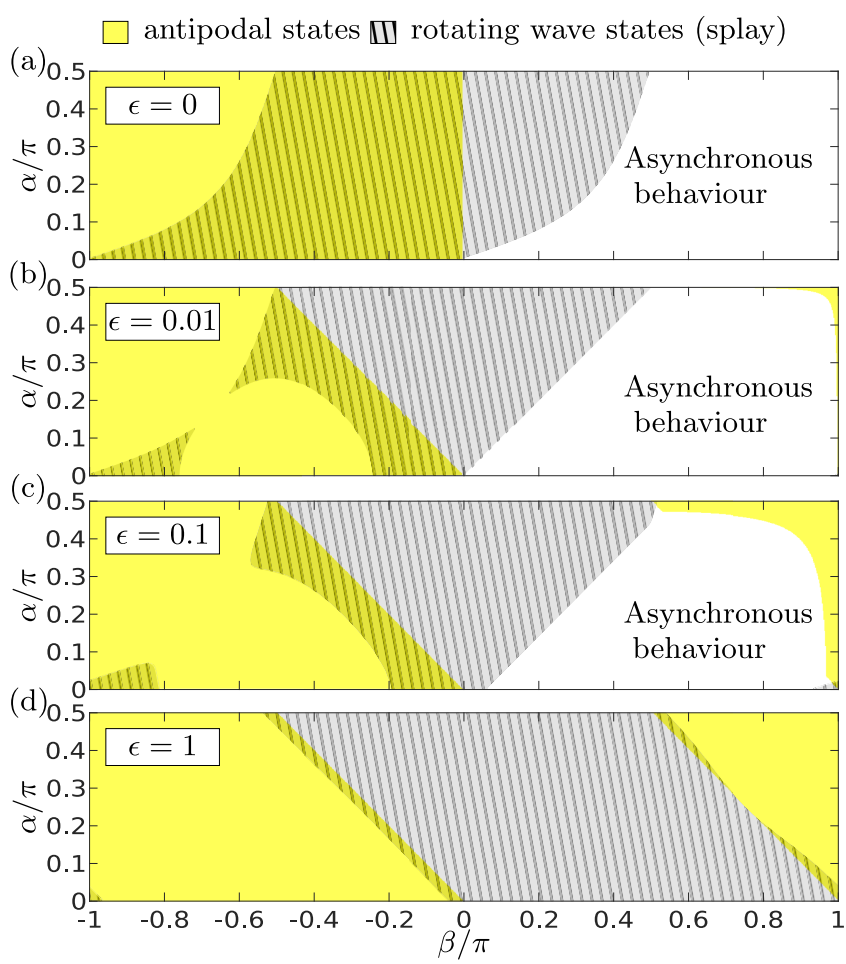

FIG. 5. The regions of stability for antipodal and rotating-wave states are presented in $(\alpha, \beta)$ parameter space for different values of $\epsilon$. Colored and hatched areas refer to stable regions for these states as indicated in the legend. White areas refer to region where these one-cluster states are unstable. (a) $\epsilon=0$; (b) $\epsilon=0.01$; (c) $\epsilon=0.1$; (d) $\epsilon=1$.

we review Corollary 4.3 from Ref. 20 as Proposition 1 and provide an extension to the whole class of antipodal states, see Corollary 5. Further, all other proofs are provided in the Appendix, as well.

A linear stability analysis for double antipodal states shows that they are unstable for all parameters $\alpha$ and $\beta$, see Corollary 7 in the Appendix. The role of the double antipodal clusters is discussed further in Sec. VI.

In Fig. 5, the regions of stability are presented for several values of the time separation parameter $\epsilon$. The first case in panel (a) assumes $\epsilon=0$, where the network structure is nonadaptive but fixed to the values given by the one-cluster states, i.e., $\kappa_{i j}=-\sin \left(a_{i}-a_{j}+\beta\right)$ as given in Sec. IV. The linearized system in this case is given by

$$
\begin{aligned}
\frac{\mathrm{d} \delta \phi_{i}}{\mathrm{~d} t}= & -\frac{1}{2 N} \sum_{j=1}^{N}(\sin (\alpha-\beta) \\
& \left.-\sin \left(2\left(a_{i}-a_{j}\right)+\alpha+\beta\right)\right)\left(\delta \phi_{i}-\delta \phi_{j}\right) .
\end{aligned}
$$

For the synchronized or antipodal state, the value $2 a_{i} \bmod 2 \pi$ is the same for all $i$. Hence, the term $2\left(a_{i}-a_{j}\right)$ disappears and the linearized system (8) possesses the same form as the linearized system for the synchronized state of the Kuramoto-Sakaguchi system ${ }^{43}$ with coupling constant $\sigma(\beta)=-\sin (\beta)$. As it follows from Ref. 43, the synchronized as well as all other antipodal states are stable for $\sigma(\beta) \cos (\alpha)>0$. The region of stability of the rotating-wave cluster has a more complex shape, see hatched area in Fig. 5(a). We find large areas where both types of one-cluster states are stable simultaneously, as well as the regions where no frequency synchronized state is stable. The results shown in Fig. 5(a) are in agreement with Ref. 12, where the authors consider the case $\epsilon=0$ in order to approximate the limit case of extremely slow adaptation $\epsilon \rightarrow 0$. However, such an approach for studying the stability of clusters for small adaptation is not correct in general. As Figs. 5(b)-5(d) show, the stability of the network with small adaptation $\epsilon>0$ is different.

The case $\epsilon=0.01$ is shown in Fig. 5(b), where we observe regions for stable antipodal and rotating-wave states as well. The introduction of a small but nonvanishing adaptation changes the regions of stability significantly. The diagram in Fig. 5(b) remains qualitatively the same for smaller values of $\epsilon$. This can be read off from the analytic findings presented in the Proposition 1 and Corollary 5. The changes in the stability areas are due to subtle changes in the equation which determine the eigenvalue of the corresponding linearized system. In fact, the adaptation introduces the necessary condition $\sin (\alpha+\beta)<0$ for the stability of antipodal states. Additionally, for all splay states, including rotating-wave states, the necessary condition $\sin (\alpha-\beta)+2 \epsilon>0$ is introduced, see Proposition 8 . This is why one can observe a nontrivial effect of adaptivity on the stability in Fig. 5. In particular, the parameter $\beta$, which determines the plasticity rule, has now a nontrivial impact on the stability of antipodal states for any $\epsilon>0$. As can be seen in Fig. 5(b), one-cluster states of antipodal type are supported by a Hebbianlike adaption $(\beta \approx-\pi / 2)$, while splay states are supported by causal rules $(\beta \approx 0)$. For the asynchronous region, the dynamical system (1)-(2) can exhibit very complex dynamics and show chaotic motion. ${ }^{13}$ This region is supported by an anti-Hebbian-like rule $(\beta \approx \pi / 2)$.

By increasing the parameter $\epsilon$, see Figs. 5(c) and 5(d), two observations can be made. First, the region of asynchronous dynamical behavior is shrinking. For $\epsilon=1$, we find at least one stable onecluster state for any choice of the phase lag parameters $\alpha$ and $\beta$. Second, the regions where both types of one-cluster states are stable are shrinking as well. In the limit of instant network adaptation, i.e., $\epsilon \rightarrow \infty$, the stability regions are completely separated. Both types of one-cluster states divide the whole parameter space into two areas. In this case, the boundaries are described by $\alpha+\beta=0$ and $\alpha+\beta=\pi$. This division can be seen from the analytic findings presented in Proposition 1 (Appendix). In the case of antipodal states, the quadratic equation which determines the Lyapunov coefficients has negative roots if and only if $\epsilon+\cos (\alpha) \sin (\beta)>0$ and $\sin (\alpha+\beta)<0$. Here, even for $\epsilon>1$, the condition $\sin (\alpha+\beta)<0$ is the only remaining one. Similarly, we find $\sin (\alpha+\beta)>0$ as a condition for the stability of rotating-wave states for $\epsilon \rightarrow \infty$.

\section{DOUBLE ANTIPODAL STATES}

Splay and antipodal clusters serve as building blocks for multicluster states. The third type, the double antipodal clusters, are not of this nature since they appear to be unstable everywhere. As unstable objects, they can still play an important role in the dynamics. Here, we 
would like to present an example, where the double antipodal clusters become part of a simple heteroclinic network. As a result, they can be observed as metastable states in numerics.

As an example, we first analyze the system of $N=3$ adaptively coupled phase oscillators which is the smallest system with a double antipodal state. According to the definition of a double antipodal state, laid out in Sec. IV, the phases $a_{i}$ of the oscillators $\phi_{i}$ are allowed to take values from the set $\{0, \pi, \psi, \psi+\pi\}$ where $\psi$ uniquely solves Eq. (6). Further, at least one oscillator $\phi_{i}$ with $a_{i} \in\{0, \pi\}$ and one oscillator $\phi_{j}(j \neq i)$ with $a_{j} \in\{\psi, \psi+\pi\}$ are needed in order to represent one of the two antipodal groups. Note that for the parameters given in Fig. 6 and $N=3$, Eq. (6) yields $\psi=1.602 \pi$ if $a_{1}, a_{2} \in\{0, \pi\}$ and $a_{3} \in\{\psi, \psi+\pi\}$.

In Fig. 6(a), we present trajectories which initially start close to antipodal clusters. The trajectories in phase space are represented by the relative coordinates $\theta_{12}=\phi_{1}-\phi_{2}$ and $\theta_{13}=\phi_{1}-\phi_{3}$. In particular, the two configurations with $\left(\theta_{12}=0, \theta_{13}=0\right)$ and $\left(\theta_{12}=0, \theta_{13}=\right.$ $\pi$ ) are considered. The coupling weights are initialized according to Eq. (5). With the given parameters, the unstable manifold of the antipodal state is one-dimensional which can be determined via Proposition 1. For the numerical simulation, we perturb the antipodal state in such a way that two distinct orbits close to the unstable manifold are visible. For both configurations, two orbits are displayed in Fig. 6(a). It can be observed that, after leaving the antipodal state, the trajectories approach the double antipodal states before leaving it toward the direction of a splay state. With this, we numerically find orbits close to "heteroclinic," which connect antipodal, double antipodal, and splay clusters, see the schematic picture on the right in Fig. 6(b). The phase differences $\theta_{12}$ and $\theta_{13}$ at the double antipodal state agree with the solution $\psi$ of Eq. (6) or $\psi+\pi$. Figure 6 (b) further justifies our statements on the heteroclinic contours. Here, we see the time series for the second moment order parameter for all trajectories in Fig. 6(b). It can be seen that in all cases we start at an antipodal cluster $\left(R_{2}(\phi)=1\right)$ from which the double antipodal state $\left[R_{2}(\phi) \approx 0.447\right.$, theoretical] is quickly approached. The trajectories stay close to the double antipodal cluster for approximately 2000 time units (shaded area) before leaving the invariant set toward the splay state $\left[R_{2}(\phi)=0\right]$.

As a second example, we analyze a system of $N=100$ adaptively coupled phase oscillators. Here, we choose two particular antipodal states as the initial condition and add a small perturbation to both. One of the states is chosen as an in-phase synchronous cluster. In both cases, the couplings weights are initialized in accordance with Eq. (5). For Fig. 6(c), we depict the trajectories which show a clear heteroclinic contour between antipodal, double antipodal, and splay state as in the example of three phase oscillators. We illustrate the heteroclinic connections and present $R_{2}(t)$ for the corresponding trajectories in Fig. 6(c). Here, the zoomed view clearly shows that the trajectories for both initial conditions starting at an antipodal cluster $\left(R_{2}(\phi)=1\right)$ again first approach a double antipodal state $\left[R_{2}(\phi) \approx 0.990\right.$, theoretical] before leaving it toward a splay state $\left(R_{2}(\phi)=0\right)$. More precisely, each trajectory comes close to a particular double antipodal state for which only one oscillator has a phase in $\{\psi, \psi+\pi\}$. Remarkably, these states, also known as solitary states, have been found in a range of other systems of coupled oscillators, as well. With Proposition 4 in the Appendix, one can show that these double antipodal states have a stable manifold with codimension one which

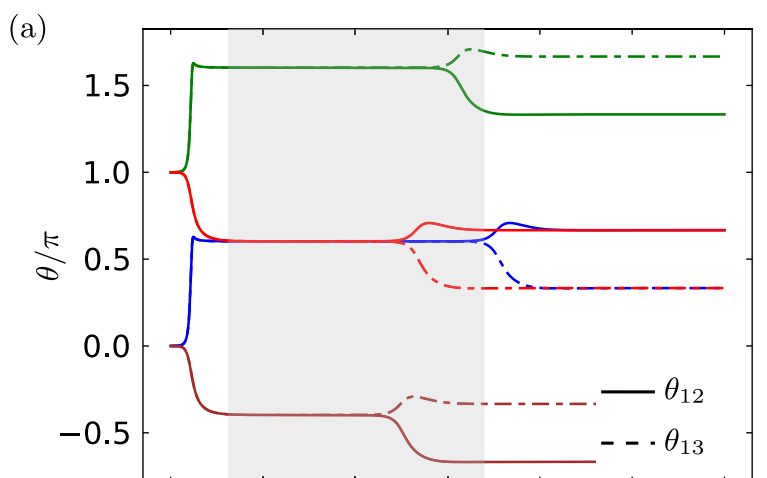

(b)
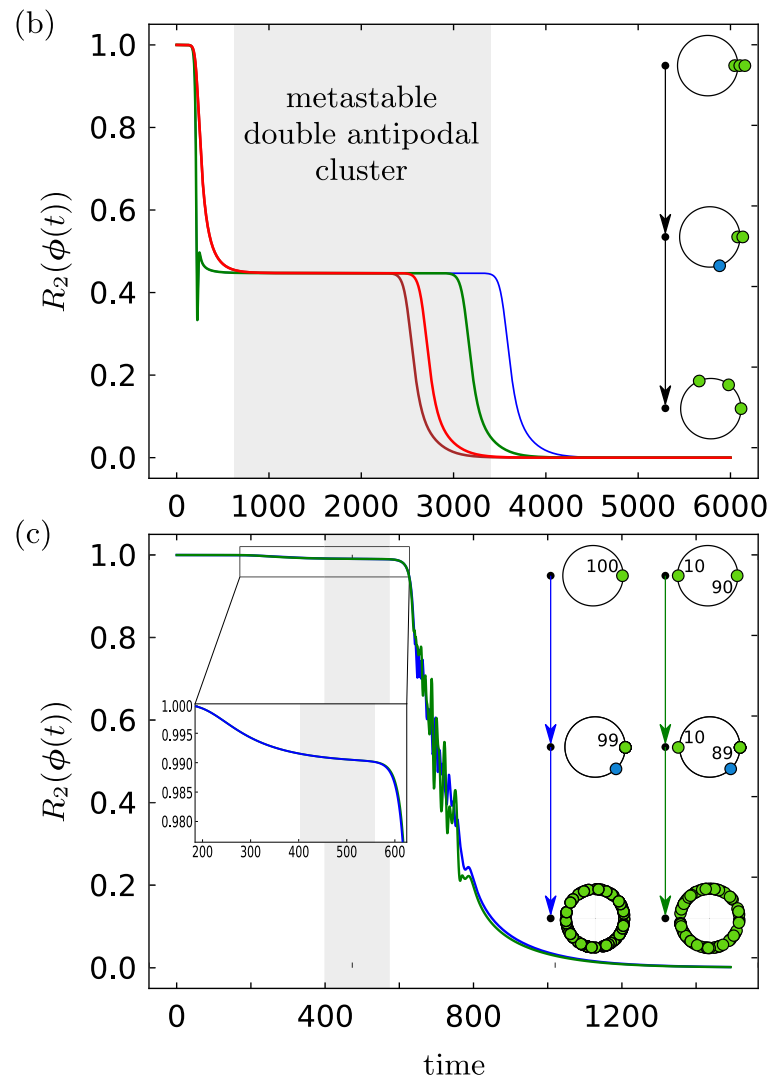

FIG. 6. Heteroclinic orbits between several steady states in a system of 3 and 100 adaptively coupled phase oscillators. (a) The time series for the relative phases $\theta_{12}$ (solid lines) and $\theta_{13}$ (dashed lines) for $N=3$ are shown. Lines with the same color correspond to the same trajectories. Panels (b) and (c) show time series for the second moment order parameter $R_{2}(\phi(t))$ as well as a schematic illustration of the observed heteroclinic connections (right) for (b) $N=3$ and (c) $N=100$. Parameter values: $\epsilon=0.01, \alpha=0.4 \pi$, and $\beta=-0.15 \pi$.

thus divides the phase space. Next to this fact, numerical evidence for the existence of heteroclinic connections between antipodal and double antipodal states as well as between double antipodal and the family of splay states is provided in Fig. 6(c). With this, double 
antipodal states play an important role in the organization of the dynamics in system (1) and (2).

\section{EMERGENCE OF MULTICLUSTER STATES AND THE ROLE OF THE TIME-SEPARATION PARAMETER}

In this section, we show the importance of the time-separation parameter $\epsilon$ for the appearance of multicluster states. In particular, we obtain the critical value $\epsilon_{c}$ above which the multicluster states cease to exist. In order to shed light on the nature of multicluster states which are built out of one-cluster states, we review the following analytic result for two-cluster states of splay type which was explicitly derived in Ref. 20. Suppose we have two groups of splay cluster states, i.e., $R_{2}\left(\mathbf{a}_{\mu}\right)=0$ for $\mu=1,2$. Then their combination leads to the following two-cluster solution of the system (1) and (2):

$$
\begin{aligned}
\phi_{i, 1}(t) & =\Omega_{1} t+a_{i, 1}, \quad i=1, \ldots, N_{1}, \\
\phi_{i, 2}(t) & =\Omega_{2} t+a_{i, 2}, \quad i=1, \ldots, N_{2}, \\
\kappa_{i j, \mu \mu}(t) & =-\sin \left(a_{i, \mu}-a_{j, \mu}+\beta\right), \quad \mu=1,2, \\
\kappa_{i j, \mu \nu}(t) & =-\rho_{\mu \nu} \sin \left(\Delta \Omega_{\mu \nu} t+a_{i, \mu}-a_{j, v}+\beta-\psi_{\mu \nu}\right),
\end{aligned}
$$

where $\mu \neq v, \Delta \Omega_{\mu \nu}:=\Omega_{\mu}-\Omega_{v}, \psi_{\mu \nu}:=\arctan \left(\Delta \Omega_{\mu \nu} / \epsilon\right)$,

$$
\begin{gathered}
\rho_{\mu \nu}:=\left(1+\left(\Delta \Omega_{\mu \nu} / \epsilon\right)^{2}\right)^{-\frac{1}{2}}, \\
\Omega_{\mu}=\frac{1}{2}\left(n_{\mu} \cos (\alpha-\beta)+\rho_{\mu \nu}\left(1-n_{\mu}\right) \cos \left(\alpha-\beta+\psi_{\mu \nu}\right)\right), \\
\left(\Delta \Omega_{12}\right)_{1,2}=\frac{1}{2}\left(n_{1}-\frac{1}{2}\right) \cos (\alpha-\beta) \\
\pm \frac{1}{2} \sqrt{\left(n_{1}-\frac{1}{2}\right)^{2} \cos ^{2}(\alpha-\beta)-2 \epsilon(2 \epsilon+\sin (\alpha-\beta)),}
\end{gathered}
$$

and $n_{1}:=N_{1} / N$.

It is quite remarkable that, in the case of splay-type clusters, the multicluster solution can be explicitly given. For a proof, we refer the reader to Ref. 20.

With this, we can study directly the role of several parameters for the existence of multicluster states. In Fig. 7, solutions for Eq. (9) are presented depending on the parameter $\beta$. The number of oscillators in the system is chosen as $N=50$. Each line $\Delta \Omega_{12}(\beta)$ in Fig. 7 represents a frequency difference of two clusters for which the two-cluster state of splay type exists with fixed relative number $n_{1}$ of oscillators in the first cluster. Note that the number of possible two-cluster states increases proportionally to the total number of oscillators $N$. Different panels show solutions for different values of $\epsilon$. We note that the existence of those two-cluster states depends only on the difference of $\gamma:=\alpha-\beta$, see Eq. (9). The necessary condition for the existence of a two-cluster state reads

$$
\left(n_{1}-\frac{1}{2}\right)^{2} \cos ^{2} \gamma>2 \epsilon(2 \epsilon+\sin \gamma) .
$$

From Eq. (10), we immediately see that the value of the time separation parameter $\varepsilon$ in system (1) and (2) is important for the existence of the multicluster states. This dependence is in contrast to the findings for one-cluster states. First of all, note that the left hand side of condition (10) is positive for $\gamma \neq \pm \pi / 2$. Hence, for all parameters, there is a critical value $\epsilon_{c}$ such that there exists no two-cluster state for $\epsilon>\epsilon_{c}$. Explicitly, we have

$$
\epsilon_{c}=-\frac{1}{4} \sin \gamma+\frac{1}{2} \sqrt{\frac{1}{4} \sin ^{2} \gamma+\left(n_{1}-\frac{1}{2}\right)^{2} \cos ^{2} \gamma},
$$

which is illustrated in Fig. 8. The figure shows the critical value $\epsilon_{c}$ depending on the parameter $\gamma$ for different values of $n_{1}$. The function possesses a global maximum with $\epsilon_{c}=0.5$. This means that there is a particular requirement on the time separation in order to have twocluster states of splay type. Indeed, the adaptation of the network has to be at most half as fast as the dynamics of the oscillatory system.

Further let us remark that the two-cluster state with equallysized clusters $n_{1}=0.5$ exists only for $\alpha-\beta \in(\pi, 2 \pi)$, i.e., $\epsilon_{c}=0$ for all $\alpha-\beta \in[0, \pi]$.

In Sec. III, we discussed that the combination of one-cluster states to a multicluster state can result in modulated dynamics of the oscillators additional to the linear growth. In fact, this additional temporal behavior is due to the interaction of the clusters. As we see in Fig. 3(a), oscillators interacting with a splay-type cluster will not be forced to perform additional dynamics. This is the reason why we are able to derive a closed analytic expression for multicluster states of splay type. It is possible to determine the frequencies explicitly as in Eq. (9). Therefore, here the interaction between cluster causes only

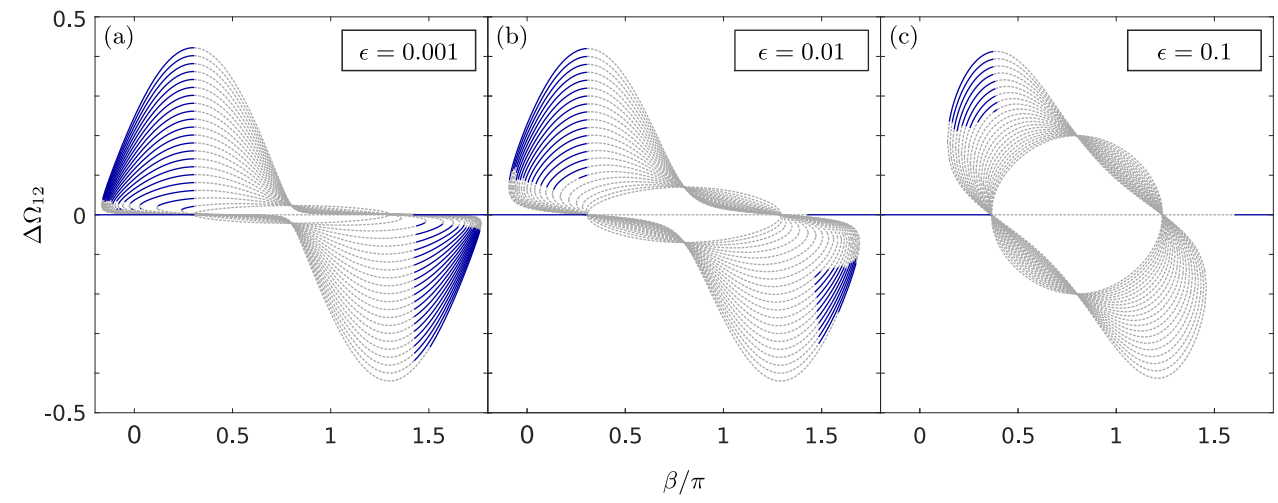

FIG. 7. All possible one- and two-cluster solutions of splay type of system (1) and (2). For fixed relative size of the first cluster $n_{1}$, the frequency differences $\Delta \Omega_{12}(\beta)$ are displayed as a function of the system parameter $\beta$ corresponding to Eq. (9). The dashed lines (gray) indicate unstable solutions while the solid lines (blue) indicate stable solutions. Parameter values $N=50$ and $\alpha=0.3 \pi$ are fixed for all panels. 


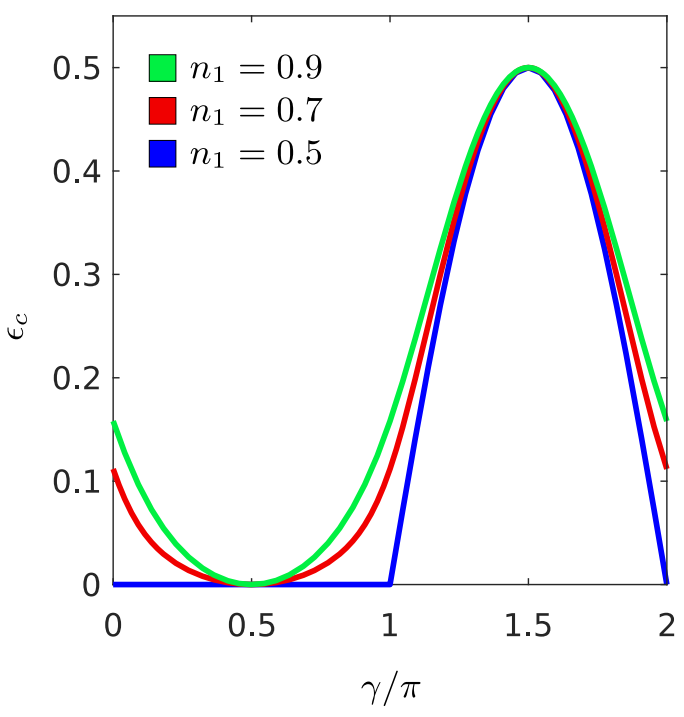

FIG. 8. For the case of two-cluster states of splay type, the critical value $\epsilon_{\mathrm{c}}$ of time-separation parameter $\epsilon$ is plotted as a function of $\gamma=\alpha-\beta$ for different cluster sizes $n_{1}=N_{1} / N$. The function is given explicitly by Eq. (11).

changes in the collective frequencies which are small whenever $\epsilon$ is small.

In contrast to splay clusters, the interaction with antipodal clusters leads to bounded modulation of the oscillator dynamics besides the constant-frequency motion. The modulations scale with $\epsilon$ and hence depend on the time-separation parameter. More rigorous results can be found in Ref. 20. In the case of a mixed type two-cluster state, both interaction phenomena are present. The oscillators in the antipodal cluster interact with the splay cluster leading to no additional modulation. On the contrary, the phase oscillators of the splay cluster get additional modulation via the interaction with the antipodal cluster.

The one-cluster states of any size apparently serve as building blocks for multicluster states. However, not all possible multicluster states are stable even though the building blocks are. Sec. VIII is devoted to this question of stability.

\section{STABILITY OF MULTICLUSTER STATES}

As mentioned above, the stability of one-cluster states is important for the stability of multicluster states. For two weakly coupled clusters, the stability of one-clusters serves as a necessary condition for the stability of the two-cluster state. In Fig. 7, the possible two-clusters of splay type are plotted. In addition, for each of these solutions, stability is analyzed numerically. For this, we initialize the system (1) and (2) on the corresponding two-cluster state and run the simulation for $t=10000$ time units. After the simulation, we compare the initial condition with the final state in order to determine stability. For each parameter value $\beta$, we color the line blue (solid line) whenever the two-cluster state is stable. Otherwise, the line is gray (dashed line). An additional line at $\Delta \Omega_{12}=0$ is plotted corresponding to the one-cluster solution. The stability of the onecluster solution is determined analytically as in Fig. 5. We note that for, $\epsilon=0.001$, a stable two-cluster state exists for almost every relative cluster size $n_{1}$, while this is not true for $\epsilon=0.01$ and even more so for $\epsilon=0.1$.

Another observation from Figs. 7(a) and 7(b) is that the possible $\beta$-values where the two-cluster states can be stable mainly correspond to the $\beta$ values where the one-cluster state is stable. This is true for small values of $\epsilon$, however, a careful inspection of Fig. 7(c) for the case of larger $\epsilon$, here $\epsilon=0.1$, shows that some two-cluster states appear to be stable for a parameter region where the corresponding one-cluster state is unstable. This can be explained as follows. According to (1), in the case of one-cluster states, the intracluster interactions are summed over all $N$ oscillators of the whole system. Additionally, the interactions are scaled with the factor $1 / N$. Therefore, the total interaction scales with 1 . For two-cluster states, the intracluster interactions for each individual cluster are only a sum over the $N_{\mu}(\mu=1,2)$ oscillators, whereas the scaling remains $1 / N$. Hence, the total intracluster interaction scales with $n_{\mu}=N_{\mu} / N$, the relative size of the cluster. Therefore, the effective oscillatory system, when neglecting the interaction to the other cluster, reads

$$
\begin{aligned}
\frac{\mathrm{d} \phi_{i, \mu}}{\mathrm{d} t} & =-\frac{n_{\mu}}{N_{\mu}} \sum_{j=1}^{N_{\mu}} \kappa_{i j, \mu \mu} \sin \left(\phi_{i, \mu}-\phi_{j, \mu}+\alpha\right), \\
\frac{\mathrm{d} \kappa_{i j, \mu \mu}}{\mathrm{d} t} & =-\epsilon\left(\kappa_{i j, \mu \mu}+\sin \left(\phi_{i, \mu}-\phi_{j, \mu}+\beta\right)\right) .
\end{aligned}
$$

This system is equivalent to (1) and (2) with $N_{\mu}$ oscillators by rescaling $\epsilon \mapsto \epsilon / n_{\mu}$. Thus, the stability of the intracluster system has to be evaluated with respect to the rescaled effective parameter $\epsilon_{\text {eff }}:=$ $\epsilon / n_{\mu}$. Since $n_{\mu}<1$ for $\mu=1,2$, we have $\epsilon_{\text {eff }}>\epsilon$. As we have discussed, stability for the one-cluster changes with increasing $\epsilon$. With this, the influence of the cluster size as well as the slight boundary shift in the regions of stability, see Fig. 7, can be explained.

Finally, we note why the equally-sized splay-clusters are not found to be stable. Indeed, from Eq. (9) we know that $2 \epsilon+\sin$ $(\alpha-\beta)<0$ is a necessary condition to have such equally-sized $\left(n_{1}=1 / 2\right)$ clusters. However, any one-cluster splay state is unstable for $2 \epsilon+\sin (\alpha-\beta)<0$ by Proposition 8 in the Appendix.

\section{CONCLUSION}

In summary, we have studied a paradigmatic model of adaptively coupled phase oscillators. It is well known that various models of weakly coupled oscillatory systems can be reduced to coupled phase oscillators. Our study has revealed the impact of synaptic plasticity upon the collective dynamics of oscillatory systems. For this, we have implemented a simplified model which is able to describe the slow adaptive change of the network depending on the oscillatory states. The slow adaptation is controlled by a time scale separation parameter.

We have described the appearance of several different frequency cluster states. Starting from random initial conditions, our numerical simulations show two different types of states. These are the splay and the antipodal type multicluster states. A third mixed type multicluster state is found by using mathematical methods described in detail in Ref. 20. For all these states, the collective motion of oscillators, the 
shape of the network, and the interaction between the frequency clusters is presented in detail. It turns out that the oscillators are able to form groups of strongly connected units. The interaction between the groups is weak compared to the interaction within the groups. The analysis of multicluster states reveals the building blocks for these states.

In particular, the following three types of relative equilibria form building blocks for multicluster states: splay, antipodal, and double antipodal. In order to understand the stability of the frequency cluster states, we perform a linear stability analysis for the relative equilibria. The stability of these states is rigorously described, and the impact of all parameters is shown. We prove that the double antipodal states are unstable in the whole parameter range. They appear to be saddlepoints in the phase space which, therefore, cannot be building blocks for higher multicluster states. While the time scale separation has no influence upon their existence, it plays an important role in the stability of the relative equilibria. The regions of stability in parameter space are presented for different choices of the time scale separation parameter. The singular limit $(\epsilon \rightarrow 0)$ and the limit of instantaneous adaptation are analyzed. The latter shows that the stability region of the splay and the antipodal states divide the whole space into two equally-sized regions without intersection. Instantaneous adaption cancels multistability of these states. The consideration of the singular limit shows that it differs from the case of no adaptation. Therefore, even for very slow adaptation, the oscillatory dynamics alone is not sufficient to describe the stability of the system.

Subsequently, the role of double antipodal states is discussed. We find that, in a system of 3 oscillators, these states are transient states in a small heteroclinic network between antipodal and splay states. They appear to be metastable, i.e., observable for a relatively long time and, therefore, are physically important transient states. Moreover, an additional analysis for an ensemble of 100 phase oscillators has revealed the importance of the double antipodal states for the global dynamics of the whole system.

For the splay clusters, we analytically show the existence of two-cluster states. Remarkably, while the existence of the one-cluster states does not depend on the time scale separation parameter, the multicluster states crucially depend on the time scale separation. In fact, we provide an analysis showing that there exists a critical value for the time scale separation. Moreover, we show that, in the case of two-cluster states of splay type, the adaption of the coupling weights must be at most half as fast as the dynamics of the oscillators. This fact is of crucial importance for comparing dynamical scenarios induced by short-term or long-term plasticity. ${ }^{46}$

The stability of two-cluster states is analyzed numerically and presented for different values of the time scale separation parameter. By assuming weakly interacting clusters, we describe the stability of the two-cluster with the help of the analysis of one-cluster states. The simulations show that there are no stable two-cluster states with clusters of the same size. We provide an argument to understand this property of the system.

\section{ACKNOWLEDGMENTS}

The authors acknowledge financial support by the Deutsche Forschungsgemeinschaft (DFG) (German Research Foundation)Project Nos. 411803875 (S.Y.) and 308748074 (R.B.). V.N. and D.K. acknowledge financial support of the Russian Foundation for Basic Research (RFBR) (Project Nos. 17-02-00874, 18-29-10040, and 1802-00406).

\section{APPENDIX: STABILITY OF ONE-CLUSTER STATES}

In order to study the local stability of one-cluster solutions described in Sec. IV, we linearize the system of differential equations (1) and (2) around the phase locked states described by $\phi_{i}=\Omega t+a_{i}$ and $\kappa_{i j}=-\sin \left(a_{i}-a_{j}+\beta\right)$. We obtain the following linearized system:

$$
\begin{aligned}
\frac{d}{d t} \delta \phi_{i}= & \frac{1}{2 N} \sum_{j=1}^{N} \sin (\beta-\alpha)\left(\delta \phi_{i}-\delta \phi_{j}\right) \\
& +\frac{1}{2 N} \sum_{j=1}^{N} \cos \left(2\left(a_{i}-a_{j}\right)+\alpha+\beta\right)\left(\delta \phi_{i}-\delta \phi_{j}\right) \\
& -\frac{1}{N} \sum_{j=1}^{N} \sin \left(a_{i}-a_{j}+\alpha\right) \delta \kappa_{i j}
\end{aligned}
$$

and

$$
\frac{d}{d t} \delta \kappa_{i j}=-\epsilon\left(\delta \kappa_{i j}+\cos \left(a_{i}-a_{j}+\beta\right)\left(\delta \phi_{i}-\delta \phi_{j}\right)\right),
$$

Note that this set of equations can be brought into the following block form:

$$
\frac{\mathrm{d}}{\mathrm{d} t}\left(\begin{array}{c}
\delta \boldsymbol{\phi} \\
\delta \kappa
\end{array}\right)=\left(\begin{array}{cc}
A & B \\
C & -\epsilon \mathbb{I}_{N^{2}}
\end{array}\right)\left(\begin{array}{l}
\delta \boldsymbol{\phi} \\
\delta \kappa
\end{array}\right)
$$

where $(\delta \boldsymbol{\phi})^{T}=\left(\delta \phi_{1}, \ldots, \delta \phi_{N}\right),(\delta \kappa)^{T}=\left(\delta \kappa_{11}, \ldots, \delta \kappa_{1 N}, \delta \kappa_{21}, \ldots\right.$, $\left.\delta \kappa_{N N}\right), B=\left(\begin{array}{lll}B_{1} & \cdots & B_{N}\end{array}\right), C=\left(\begin{array}{c}C_{1} \\ \vdots \\ C_{N}\end{array}\right)$, and $A, B_{n}, C_{n}$ are $N \times N$ matrices with $n=1, \ldots, N$. The elements of the block matrices read

$$
\begin{aligned}
& a_{i j}= \begin{cases}-\frac{1}{2} \sin (\alpha-\beta)-\frac{1}{N} \sin (\beta) \cos (\alpha) \\
+\frac{1}{2 N} \sum_{k=1}^{N} \sin \left(2\left(a_{i}-a_{k}\right)+\alpha+\beta\right), \\
\frac{1}{2 N}\left(\sin (\alpha-\beta)-\sin \left(2\left(a_{i}-a_{j}\right)+\alpha+\beta\right)\right), & i \neq j,\end{cases} \\
& b_{i j ; n}= \begin{cases}-\frac{1}{N} \sin \left(a_{n}-a_{j}+\alpha\right), & i=n, \\
0 & \text { otherwise, }\end{cases} \\
& c_{i j ; n}= \begin{cases}0, & j=n, i=j \\
-\epsilon \cos \left(a_{n}-a_{i}+\beta\right), & j=n, i \neq j, \\
\epsilon \cos \left(a_{n}-a_{i}+\beta\right), & j \neq n, i=j, \\
0 & \text { otherwise. }\end{cases}
\end{aligned}
$$

Throughout this appendix, we will make use of Schur's complement ${ }^{47}$ in order to simplify characteristic equations. In particular, any $m \times m$ 
matrix $M$ in the $2 \times 2$ block form can be written as

$$
\begin{aligned}
M & =\left(\begin{array}{ll}
A & B \\
C & D
\end{array}\right) \\
& =\left(\begin{array}{cc}
\mathbb{I}_{p} & B D^{-1} \\
0 & \mathbb{I}_{q}
\end{array}\right)\left(\begin{array}{cc}
A-B D^{-1} C & 0 \\
0 & D
\end{array}\right)\left(\begin{array}{cc}
\mathbb{I}_{p} & 0 \\
D^{-1} C & \mathbb{I}_{q}
\end{array}\right),
\end{aligned}
$$

where $A$ is a $p \times p$ matrix and $D$ is an invertible $q \times q$ matrix. The matrix $A-B D^{-1} C$ is called Schur's complement. A simple formula for the determinant of $M$ can be derived with this decomposition in (A4),

$$
\operatorname{det}(M)=\operatorname{det}\left(A-B D^{-1} C\right) \cdot \operatorname{det}(D) .
$$

This result is important for the subsequent stability analysis. Note that in the following, an asterisk indicates the complex conjugate.

Proposition A.1. Suppose we have $\mathbf{a}_{k}=\left(0, \frac{2 \pi}{N} k, \ldots,(N-1)\right.$ $\left.\frac{2 \pi}{N} k\right)^{T}$ and the characteristic equation of the linear system (A1) and (A2), then the set of eigenvalues $L$ are as follows.

1. (in-phase and antiphase synchrony) If $k=0$ or $k=N / 2$,

$$
L=\left\{(0)_{1},(-\epsilon)_{(N-1) N+1},\left(\lambda_{1}\right)_{N-1},\left(\lambda_{2}\right)_{N-1}\right\},
$$

where $\lambda_{1}$ and $\lambda_{2}$ solve $\lambda^{2}+(\epsilon-\cos (\alpha) \sin (\beta)) \lambda-\epsilon \sin$ $(\alpha+\beta)=0$.

2. (incoherent rotating-wave) If $k \neq 0, N / 2, N / 4,3 N / 4$, the spectrum is

$$
\begin{gathered}
L=\left\{(0)_{N-2},(-\epsilon)_{(N-1) N+1},\left(-\frac{\sin (\alpha-\beta)}{2}-\epsilon\right)_{N-3},\right. \\
\left.\left(\vartheta_{1}\right)_{1},\left(\vartheta_{2}\right)_{1},\left(\vartheta_{1}^{*}\right)_{1},\left(\vartheta_{2}^{*}\right)_{1}\right\},
\end{gathered}
$$

where $\vartheta_{1}$ and $\vartheta_{2}$ solve $\vartheta^{2}+\left(\epsilon+\frac{1}{2} \sin (\alpha-\beta)-\frac{1}{4} \mathrm{i} e^{\mathrm{i}(\alpha+\beta)}\right) \vartheta-$ $\frac{\epsilon}{2} \mathrm{i} e^{\mathrm{i}(\alpha+\beta)}=0$.

3. (4-rotating-wave state) If $k=N / 4,3 N / 4$, the spectrum is

$$
\begin{aligned}
L= & (0)_{N-1},(-\epsilon)_{(N-1) N+1}, \\
& \left.\left(-\frac{\sin (\alpha-\beta)}{2}-\epsilon\right)_{N-2},\left(\lambda_{1}\right)_{1},\left(\lambda_{2}\right)_{1}\right\},
\end{aligned}
$$

where $\lambda_{1}$ and $\lambda_{2}$ solve

$$
\lambda^{2}+(\epsilon+\sin (\alpha) \cos (\beta)) \lambda+\epsilon \sin (\alpha+\beta)=0 .
$$

Here, the multiplicities for each eigenvalue are given as subscripts.

Proof. The proof can be found in Ref. 20.

So far, we have found the Lyapunov coefficients for the rotatingwave states. The following two Lemmata are needed to describe the stability of antipodal, 4-phase-cluster, and double antipodal states as well.

Lemma A.2. Suppose $M$ is a block square matrix of the form

$$
M=\left(\begin{array}{cc}
A & m_{1} \hat{1}_{p, q} \\
m_{2} \hat{1}_{q, p} & B
\end{array}\right)
$$

where $A$ is a circulant $p \times p$ matrix, $B$ is a circulant $q \times q$ matrix, $\hat{1}_{p, q}$ is $p \times q$ where all entries are 1 and $m_{1}, m_{2} \in \mathbb{R}$. Then, the eigenvectoreigenvalue pairs are given by

$$
\begin{gathered}
\left(\lambda_{k}^{0}, \ldots, \lambda_{k}^{p-1}, 0, \ldots, 0\right)^{T}, \quad \mu_{k}=\sum_{l=0}^{p-1} a_{1(1+l)} \lambda_{k}^{l}, \\
\left(0, \ldots, 0, \rho_{l}^{0}, \ldots, \rho_{l}^{q-1}\right)^{T}, \quad \nu_{l}=\sum_{l=0}^{q-1} b_{1(1+l)} \rho_{l}^{l}, \\
\left(1, \ldots, 1, a_{1}, \ldots a_{1}\right)^{T}, \quad \bar{\mu}=\mu_{0}+m_{1} q a_{1}, \\
\left(1, \ldots, 1, a_{2}, \ldots, a_{2}\right)^{T}, \quad \bar{v}=\mu_{0}+m_{1} q a_{2},
\end{gathered}
$$

with $\lambda_{k}=e^{\mathrm{i} \frac{2 \pi}{p} k}$ and $\rho_{l}=e^{\mathrm{i} \frac{2 \pi}{q} l}$ for $k=1, \ldots, p-1$ and $l=1, \ldots$, $q-1$, respectively, and $a_{1}$ and $a_{2}$ solve the equation

$$
a^{2}+\frac{\mu_{0}-v_{0}}{m_{1} q} a-\frac{m_{2} p}{m_{1} q}=0
$$

with $\mu_{0}=\sum_{j=1}^{p} a_{1 j}$ and $v_{0}=\sum_{j=1}^{q} b_{1 j}$.

Proof. We can prove the Lemma by direct calculation and find

$$
\begin{aligned}
M & \left(\lambda_{k}^{0}, \ldots, \lambda_{k}^{p-1}, 0, \ldots, 0\right)^{T} \\
& =\left(A\left(\lambda_{k}^{0}, \ldots, \lambda_{k}^{p-1}\right)^{T} m_{2} \sum_{l=0}^{p-1} \lambda_{k}^{l}\right) \\
& =\mu_{k}\left(\lambda_{k}^{0}, \ldots, \lambda_{k}^{p-1}, 0, \ldots, 0\right)^{T} .
\end{aligned}
$$

Here, we use that $A$ is a circulant matrix and that $\sum_{l=0}^{p-1} \lambda_{k}^{l}=0$ for all $k=1, \ldots, p-1$. Analogous arguments hold for (A6). The last two eigenvector-eigenvalue pairs (A7) and (A8) can be obtained by

$$
\begin{aligned}
M & (1, \ldots, 1, a, \ldots, a)^{T} \\
& =\left(\begin{array}{c}
A(1, \ldots, 1)^{T}+m_{1} q a(1, \ldots, 1)^{T} \\
\frac{m_{2} p}{a}(1, \ldots, 1)^{T}+a B(1, \ldots, 1)^{T}
\end{array}\right) \\
& =\left(\begin{array}{c}
\mu_{0}+m_{1} q a \\
\frac{m_{2} p}{a}+v_{0}
\end{array}\right)(1, \ldots, 1, a, \ldots, a)^{T},
\end{aligned}
$$

which solves the eigenvalue problem if $a$ is chosen to be either $a_{1}$ or $a_{2}$.

Lemma A.3. Suppose we have a phase locked state with phases $a_{i} \in[0,2 \pi)$. Then, the solutions for the characteristic equations corresponding to the linearized system (A1) and (A2) are given by $\lambda=-\epsilon$ with multiplicity $N^{2}-N$ and by the solution of the following set of equations:

$$
\operatorname{det}\left(\left(A-\lambda \mathbb{I}_{N}\right)(\epsilon+\lambda)+B C\right)=0 .
$$

Proof. Applying Schur's decomposition (A4) to the linearized system in the block form (A3) yields the result.

Proposition A.4. Suppose we have a state with phases $a_{i} \in$ $\{0, \pi, \psi, \psi+\pi\}$ where $i=1, \ldots, N$. Further set $q_{1}=Q_{1} / N$ and $q_{2}=Q_{2} / N$, where $Q_{1}$ and $Q_{2}$ denote the numbers of phases which are 
either 0 or $\pi$ and $\psi$ or $\psi+\pi$, respectively. Then, the linear system (A1) and (A2) possesses the following set $L$ of eigenvalues:

$$
\begin{gathered}
L=\left\{(0)_{1},(-\epsilon)_{(N-1) N+1},\left(\lambda_{1}\right)_{N_{1}-1},\left(\lambda_{2}\right)_{N_{1}-1},\right. \\
\left.\left(\vartheta_{1}\right)_{N_{2}-1},\left(\vartheta_{2}\right)_{N_{2}-1},\left(\rho_{1}\right)_{1},\left(\rho_{2}\right)_{1}\right\},
\end{gathered}
$$

where $\lambda_{1}$ and $\lambda_{2}$ solve

$$
\begin{aligned}
\lambda^{2} & +\frac{1}{2}\left(\sin (\alpha-\beta)-q_{1} \sin (\alpha+\beta)\right. \\
& \left.-q_{2} \sin (-2 \psi+\alpha+\beta)+2 \epsilon\right) \lambda \\
& -\epsilon q_{1} \sin (\alpha+\beta)-\epsilon q_{2} \sin (-2 \psi+\alpha+\beta)=0,
\end{aligned}
$$

$\vartheta_{1}$ and $\vartheta_{2}$ solve

$$
\begin{aligned}
& \vartheta^{2}+\frac{1}{2}\left(\sin (\alpha-\beta)-q_{1} \sin (2 \psi+\alpha+\beta)\right. \\
& \left.\quad-q_{2} \sin (\alpha+\beta)+2 \epsilon\right) \vartheta \\
& \quad-\epsilon q_{1} \sin (2 \psi+\alpha+\beta)-\epsilon q_{2} \sin (\alpha+\beta)=0,
\end{aligned}
$$

as well as $\rho_{1}$ and $\rho_{2}$ solve

$$
\begin{aligned}
\rho^{2} & +\frac{1}{2}\left(\sin (\alpha-\beta)-q_{1} \sin (2 \psi+\alpha+\beta)\right. \\
& \left.-q_{2} \sin (-2 \psi+\alpha+\beta)+2 \epsilon\right) \rho \\
& -\epsilon q_{1} \sin (2 \psi+\alpha+\beta)-\epsilon q_{2} \sin (-2 \psi+\alpha+\beta)=0 .
\end{aligned}
$$

The multiplicities for each eigenvalue are given as subscripts.

Proof. For an arbitrary solution of the form $\phi_{i}=\Omega t+a_{i}$, we consider the linearized system (A1) and (A2) in the block form (A3) and apply Lemma A.3. The elements of the second term $D:=B C$ of Schur's complement are then

$$
d_{i j}=-\frac{\epsilon}{2 N}\left(\sin (\alpha-\beta)+\sin \left(2\left(a_{i}-a_{j}\right)+\alpha+\beta\right)\right)
$$

if $i \neq j$ and

$$
\begin{aligned}
d_{i i}= & \epsilon\left(\frac{1}{2} \sin (\alpha-\beta)-\frac{1}{N} \sin (\alpha) \cos (\beta)\right. \\
& \left.+\frac{1}{2 N} \sum_{j=1}^{N} \sin \left(2\left(a_{i}-a_{j}\right)+\alpha+\beta\right)\right) .
\end{aligned}
$$

Defining the matrix $M:=\left(A-\lambda \mathbb{I}_{N}\right)(\epsilon+\lambda)+D$ we obtain

$$
m_{i j}= \begin{cases}-\lambda^{2}+\left(a_{i i}-\epsilon\right) \lambda+\epsilon a_{i i}+d_{i i}, & i=j, \\ \lambda a_{i j}+\epsilon a_{i j}+d_{i j}, & i \neq j .\end{cases}
$$

Using the assumption for the phases $a_{i}$, then one group of oscillators (group $I$ ) have $a_{i} \in\{0, \pi\}$ and the remaining $Q_{2}$ oscillators (group $I I$ ) have $a_{i}=\{\psi, \psi+\pi\}$. Putting this into the definition of $m_{i j}$, we find that the whole square matrix $M$ can be written as

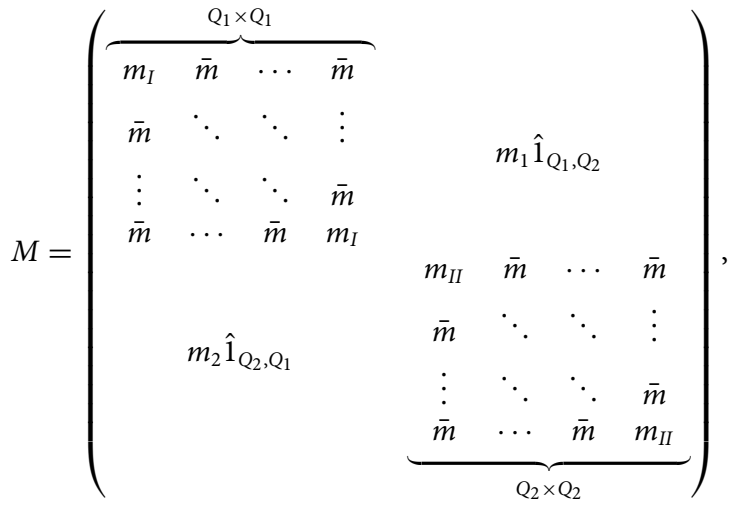

where $m_{1}, \bar{m}, m_{I}$, and $m_{I I}$ are real values which depend on all the system parameters $\alpha, \beta, \epsilon$ and additionally on $\psi$ and $\lambda$. Note that all diagonal blocks are circulant matrices. The determinant is invariant under basis transformations which is why we diagonalize the matrix $M$ and therewith derive equations for the values $\lambda$. In order to do so, we look for the eigenvalues of $M$ determined by the characteristic equation

$$
\operatorname{det}\left(M-\mu \mathbb{I}_{N}\right)=0 .
$$

Due to the structure of $M$, we can apply Lemma A.2 and find the following set of eigenvalues:

$$
\begin{aligned}
\mu_{k}= & -\lambda^{2}-\frac{1}{2}\left(\sin (\alpha-\beta)-q_{1} \sin (\alpha+\beta)-q_{2} \sin (-2 \psi+\alpha+\beta)\right. \\
& +2 \epsilon) \lambda+\epsilon q_{1} \sin (\alpha+\beta)+\epsilon q_{2} \sin (-2 \psi+\alpha+\beta),
\end{aligned}
$$

for $k=1, \ldots, Q_{1}-1$. Analogously, we obtain the equations for $v_{k}$ $\left(k=1, \ldots, Q_{2}-1\right)$ where $m_{I}$ is substituted with $m_{I I}$. The two other eigenvalue are given by $\bar{\mu}=\mu_{0}+m_{1} Q_{2} a_{1}$ and $\bar{v}=\mu_{0}+m_{1} Q_{2} a_{2}$, respectively, where

$$
\mu_{0}=m_{I}+\left(Q_{1}-1\right) \bar{m}
$$

and $a_{1,2}$ are given by

$$
a^{2}+\frac{\left(m_{I}-m_{I I}\right)+\left(Q_{1}-Q_{2}\right) \bar{m}}{m_{1} Q_{2}} a-\frac{m_{2} Q_{1}}{m_{1} Q_{2}}=0 .
$$

Considering the row sums of $M$, we find that all agree with $-\lambda^{2}-\epsilon \lambda$ and, therefore, $\bar{\mu}=-\lambda^{2}-\epsilon \lambda$. Resulting from this $a_{1}=-\left(\lambda^{2}+\epsilon \lambda+\mu_{0}\right) / m_{1} Q_{2}=1$. Hence,

$$
a_{2}=\frac{\left(m_{I I}-m_{I}\right)+\left(Q_{2}-Q_{1}\right) \bar{m}}{m_{1} Q_{2}}-1
$$

and we find

$$
\begin{aligned}
\bar{v}= & m_{I I}+\left(Q_{2}-1\right) \bar{m}-m_{1} Q_{2} \\
= & -\lambda^{2}-\frac{1}{2}\left(\sin (\alpha-\beta)-q_{1} \sin (2 \psi+\alpha+\beta)-q_{2} \sin (-2 \psi+\alpha\right. \\
& +\beta)+2 \epsilon) \lambda+\epsilon q_{1} \sin (2 \psi+\alpha+\beta)+\epsilon q_{2} \sin (-2 \psi+\alpha+\beta) .
\end{aligned}
$$

After diagonalizing the matrix $M$, the determinant can be easily written as

$$
\operatorname{det}(M)=\bar{\mu} \cdot \mu_{1} \cdots \mu_{N_{1}-1} \cdot \bar{v} \cdot v_{1} \cdots v_{N_{2}-1} .
$$


Therewith, finding $\lambda^{\prime} s$ such that at least one of the eigenvalues of $M$ vanishes solves the initial eigenvalue problem.

We will now sum up the results with the following corollaries.

Corollary A.5. The set of eigenvalues of the linearized system (A1) and (A2) around all antipodal states with $a_{i} \in\{0, \pi\}$ agrees with the set $L$ in Proposition 1 for rotating-wave states with $k=0, N / 2$.

Proof. Put $Q_{2}=0$ in Proposition 4, then there is only the equation for $\lambda$ left.

Corollary A.6. The set of eigenvalues to the linearized system (A1) and (A2) around all 4-phase-cluster states with $a_{i} \in\{0, \pi / 2, \pi, 3 \pi / 2\}$ and $R_{2}(\boldsymbol{a})=0$ agrees with the set $L$ in Proposition 1 for 4-rotating-wave states.

Proof. The requirement $R_{2}(\boldsymbol{a})=0$ yields $Q_{1}=Q_{2}$. The statement of this proposition follows by using Proposition 4 .

Corollary A.7. For all $\alpha$ and $\beta$, the double antipodal states are unstable.

Proof. Suppose the polynomial equation $p(x)=x^{2}+a x+$ $b=0$. This equation has two negative roots if and only if $b>0$ and $a>0$ meaning that $p(0)>0$ and the vertex of the parabola is at $x<$ 0 , respectively. In order to have stable double antipodal states, these two conditions have to be met by all three equations for $\lambda, \vartheta$, and $\rho$ in Proposition 4. From the condition on the existence of double antipodal states (6), we find $q_{1} \sin (2 \psi+\alpha+\beta)+q_{2} \sin (-2 \psi+\alpha+$ $\beta)=-\sin (\alpha+\beta)$. With this assumption on the quadratic equation and the latter equation, we find the following two necessary conditions for the stability of double antipodal states: (1) $q_{1} \sin (2 \psi+\alpha+$ $\beta)+q_{2} \sin (\alpha+\beta)>0$ and (2) $q_{1} \sin (2 \psi+\alpha+\beta)+q_{2} \sin (\alpha+$ $\beta)<0$. The two condition cannot be equally fulfilled.

In the following, we give a necessary condition for the stability of all one-cluster states of splay type, in contrast to the result on rotating-wave states given in Proposition 1. In general all splay onecluster states have the property $R_{2}(\boldsymbol{a})=0$ for the phase given by the vector $\boldsymbol{a}$. Therefore, the splay states form $N-2$ dimensional family of solution. Hence, around each splay states there are $N-2$ neutral variational directions $(\delta \boldsymbol{\phi}, \delta \kappa)^{T}$ which are determined by the condition $\sum_{j=1}^{N} e^{\mathrm{i} 2 a_{j}} \delta \phi_{j}=0$. Note, $\delta \kappa_{i j}=-\cos \left(a_{i}-a_{j}+\beta\right)\left(\delta \phi_{i}-\phi_{j}\right)$ in the neutral direction.

Proposition A.8. Consider an asymptotically stable one-cluster state of splay type. Then, $\epsilon+\sin (\alpha-\beta) / 2>0$.

Proof. Due to the block form of the linearized equation (A3) and the Schur decomposition (A4), any eigenvalue comes with a second. We have already seen this in Lemma A.2 and Proposition 4. Variation along the neutral direction gives $N-2$ times the eigenvalue 0 . Suppose we have $\delta \phi$ such that $\sum_{j=1}^{N} e^{\mathrm{i} 2 a_{j}} \delta \phi_{j}=0$ and $\delta \kappa_{i j}=-\cos \left(a_{i}-a_{j}+\beta\right)\left(\delta \phi_{i}-\phi_{j}\right)$. Applying Schur decomposition (A4), we get

$$
\begin{aligned}
(M- & \lambda \mathbb{I})_{N^{2}+N}\left(\begin{array}{l}
\delta \boldsymbol{\phi} \\
\delta \kappa
\end{array}\right) \\
= & \left(\begin{array}{cc}
\mathbb{I}_{N} & -(\epsilon+\lambda) B \\
0 & \mathbb{I}_{N^{2}}
\end{array}\right)\left(\begin{array}{cc}
\left(A-\lambda \mathbb{I}_{N}\right)+\frac{1}{\epsilon+\lambda} B C & 0 \\
0 & -(\epsilon+\lambda)
\end{array}\right) \\
& \times\left(\begin{array}{c}
\delta \phi \\
-\frac{1}{\epsilon+\lambda} C \delta \phi+\delta \kappa
\end{array}\right)=0 .
\end{aligned}
$$

With this, we have to find $\lambda$ such that the last equality in (A9) is fulfilled. This is equivalent to solving $\left(\left(A-\lambda \mathbb{I}_{N}\right)(\epsilon+\lambda)+B C\right) \delta \boldsymbol{\phi}=0$ of which in general only $N-2$ equations are linearly independent. The equivalence can be seen by multiplying $\epsilon+\lambda$ from both sides and keeping in mind that $\delta \kappa$ is already determined by $\delta \boldsymbol{\phi}$. Using the definition of $\delta \boldsymbol{\phi}$, the matrices $A$ and $B C$ can be effectively reduced in such a way that they are independent of the actual values for the phases $a_{j}$. In fact,

$$
\begin{aligned}
& a_{i j}= \begin{cases}-\frac{N-1}{2 N} \sin (\alpha-\beta), & i=j, \\
\frac{1}{2 N} \sin (\alpha-\beta), & i \neq j,\end{cases} \\
& (b c)_{i j}= \begin{cases}\epsilon \frac{N-1}{2 N} \sin (\alpha-\beta), & i=j, \\
-\frac{\epsilon}{2 N} \sin (\alpha-\beta), & i \neq j .\end{cases}
\end{aligned}
$$

In turn, this gives $\left(\left(A-\lambda \mathbb{I}_{N}\right)(\epsilon+\lambda)+B C\right)$ a circulant structure which can be used to diagonalize the matrix, in analogy to Proposition 4 . For circulant matrices, we immediately know the eigenvalues. They are

$$
\begin{aligned}
\mu_{l}= & -\lambda^{2}-\left(\frac{N-1}{2 N} \sin (\alpha-\beta)\right. \\
& \left.-\frac{1}{2 N} \sin (\alpha-\beta)\left(\sum_{k=0}^{N-1} e^{\mathrm{i} 2 \pi k l / N}-1\right)+\epsilon\right) \lambda,
\end{aligned}
$$

with $l=0, \ldots, N-1$ and $\operatorname{det}\left(\left(A-\lambda \mathbb{I}_{N}\right)(\epsilon+\lambda)+B C\right)=\mu_{0}(\lambda) \cdots$ $\mu_{N-1}(\lambda)$. Remember, we have, in general, $N-2$ independent equations. Thus, solving $\mu_{l}(\lambda)=0$ for $\lambda$ results in $N-2$ eigenvalues $\lambda=0,1$ eigenvalue $\lambda=-\epsilon$ and $N-3$ eigenvalues $\lambda=-\epsilon-$ $\sin (\alpha-\beta) / 2$. Note that for 4 -phase-cluster states, as considered in Corollary 6 , the number of independent equations is $N-1$. This is due to the fact that, in this case, the equations for the imaginary and real parts from $\sum_{j=1}^{N} e^{\mathrm{i} 2 a_{j}} \delta \phi_{j}=0$ agree.

\section{REFERENCES}

${ }^{1}$ T. Gross and B. Blasius, “Adaptive coevolutionary networks: A review," J. R. Soc. Interface 5, 259-271 (2008).

${ }^{2}$ W. Gerstner, R. Kempter, J. L. von Hemmen, and H. Wagner, “A neuronal learning rule for sub-millisecond temporal coding," Nature 383, 76-78 (1996).

${ }^{3}$ C. Clopath, L. Büsing, E. Vasilaki, and W. Gerstner, "Connectivity reflects coding: A model of voltage-based STDP with homeostasis," Nat. Neurosci. 13, 344-352 (2010).

${ }^{4}$ W. Gerstner, W. M. Kistler, R. Naud, and L. Paninski, Neuronal Dynamics: From Single Neurons to Networks and Models of Cognition (Cambridge University Press, 2014).

${ }^{5}$ H. Markram, J. Lübke, and B. Sakmann, "Regulation of synaptic efficacy by coincidence of postsynaptic APs and EPSPs," Science 275, 213-215 (1997).

${ }^{6}$ L. F. Abbott and S. Nelson, "Synaptic plasticity: Taming the beast," Nat. Neurosci. 3, 1178-1183 (2000).

${ }^{7}$ N. Caporale and Y. Dan, "Spike timing-dependent plasticity: A Hebbian learning rule,” Annu. Rev. Neurosci. 31, 25-46 (2008).

${ }^{8}$ L. Lücken, O. Popovych, P. Tass, and S. Yanchuk, "Noise-enhanced coupling between two oscillators with long-term plasticity," Phys. Rev. E 93, 032210 (2016). ${ }^{9} \mathrm{~S}$. Jain and S. Krishna, "A model for the emergence of cooperation, interdependence, and structure in evolving networks," Proc. Natl. Acad. Sci. U.S.A. 98, 543-547 (2001). 
${ }^{10}$ D. V. Kasatkin, S. Yanchuk, E. Schöll, and V. I. Nekorkin, "Self-organized emergence of multi-layer structure and chimera states in dynamical networks with adaptive couplings," Phys. Rev. E 96, 062211 (2017).

${ }^{11} \mathrm{~T}$. Aoki and T. Aoyagi, "Co-evolution of phases and connection strengths in a network of phase oscillators," Phys. Rev. Lett. 102, 034101 (2009).

${ }^{12} \mathrm{~T}$. Aoki and T. Aoyagi, "Self-organized network of phase oscillators coupled by activity-dependent interactions," Phys. Rev. E 84, 066109 (2011).

${ }^{13}$ D. V. Kasatkin and V. I. Nekorkin, "Dynamics of the phase oscillators with plastic couplings," Radiophys. Quant. Electron. 58, 877-891 (2016).

${ }^{14}$ V. I. Nekorkin and D. V. Kasatkin, "Dynamics of a network of phase oscillators with plastic couplings,” AIP Conf. Proc. 1738, 210010 (2016).

${ }^{15}$ A. Gushchin, E. Mallada, and A. Tang, "Synchronization of phase-coupled oscillators with plastic coupling strength," in Information Theory and Applications Workshop (IEEE, 2015), pp. 291-300.

${ }^{16} \mathrm{C}$. B. Picallo and H. Riecke, "Adaptive oscillator networks with conserved overall coupling: Sequential firing and near-synchronized states," Phys. Rev. E 83, 036206 (2011).

${ }^{17}$ L. Timms and L. Q. English, "Synchronization in phase-coupled Kuramoto oscillator networks with axonal delay and synaptic plasticity," Phys. Rev. E 89, 032906 (2014).

${ }^{18} \mathrm{Q}$. Ren and J. Zhao, "Adaptive coupling and enhanced synchronization in coupled phase oscillators," Phys. Rev. E 76, 016207 (2007).

${ }^{19}$ V. Avalos-Gaytán, J. A. Almendral, I. Leyva, F. Battiston, V. Nicosia, V. Latora, and S. Boccaletti, "Emergent explosive synchronization in adaptive complex networks," Phys. Rev. E 97, 042301 (2018).

${ }^{20}$ R. Berner, E. Schöll, and S. Yanchuk, "Multi-clusters in networks of adaptively coupled phase oscillators," SIAM J. Appl. Dyn. Syst. (to be published); e-print arXiv: 1809.00573.

${ }^{21}$ O. V. Popovych, M. N. Xenakis, and P. A. Tass, "The spacing principle for unlearning abnormal neuronal synchrony," PLoS One 10, 1-24 (2015).

${ }^{22}$ D. S. Bassett, E. Bullmore, B. A. Verchinski, V. S. Mattay, D. R. Weinberger, and A. Meyer-Lindenberg, "Hierarchical organization of human cortical networks in health and schizophrenia," J. Neurosci. 28, 9239-9248 (2008).

${ }^{23}$ D. S. Bassett, D. L. Greenfield, A. Meyer-Lindenberg, D. R. Weinberger, S. W. Moore, and E. T. Bullmore, "Efficient physical embedding of topologically complex information processing networks in brains and computer circuits," PLoS Comput. Biol. 6, el000748 (2010).

${ }^{24}$ C. Lohse, D. S. Bassett, K. O. Lim, and J. M. Carlson, "Resolving anatomical and functional structure in human brain organization: Identifying mesoscale organization in weighted network representations," PLoS Comput. Biol. 10, e1003712 (2014).

${ }^{25}$ R. F. Betzel, J. D. Medaglia, L. Papadopoulos, G. L. Baum, R. Gur, R. Gur, D. Roalf, T. D. Satterthwaite, and D. S. Bassett, "The modular organization of human anatomical brain networks: Accounting for the cost of wiring," Netw. Neurosci. 1, 42-68 (2017).

${ }^{26}$ A. Ashourvan, Q. K. Telesford, T. Verstynen, J. M. Vettel, and D. S. Bassett, "Multi-scale detection of hierarchical community architecture in structural and functional brain networks," PLoS One 14, e0215520 (2019).

${ }^{27} \mathrm{H}$. Sakaguchi and Y. Kuramoto, "A soluble active rotator model showing phase transitions via mutual entertainment," Prog. Theor. Phys. 76, 576-581 (1986).
${ }^{28}$ J. A. Acebrón, L. L. Bonilla, C. J. P. Vicente, F. Ritort, and R. Spigler, "The Kuramoto model: A simple paradigm for synchronization phenomena," Rev. Mod. Phys. 77, 137 (2005).

${ }^{29}$ Y. Kuramoto, Chemical Oscillations, Waves and Turbulence (Springer-Verlag, Berlin,1984).

${ }^{30} \mathrm{O}$. E. Omel'chenko and M. Wolfrum, "Nonuniversal transitions to synchrony in the Sakaguchi-Kuramoto model,” Phys. Rev. Lett. 109, 164101 (2012).

${ }^{31}$ S. H. Strogatz, "From Kuramoto to Crawford: Exploring the onset of synchronization in populations of coupled oscillators," Physica D 143, 1-20 (2000).

${ }^{32}$ A. Pikovsky and M. G. Rosenblum, "Partially integrable dynamics of hierarchical populations of coupled oscillators," Phys. Rev. Lett. 101, 264103 (2008).

${ }^{33}$ D. Hebb, The Organization of Behavior: A Neuropsychological Theory (Wiley, New York, 1949)

${ }^{34} \mathrm{~F}$. C. Hoppensteadt and E. M. Izhikevich, "Synaptic organizations and dynamical properties of weakly connected neural oscillators II. Learning phase information," Biol. Cybern. 75, 129-135 (1996).

${ }^{35}$ P. Seliger, S. C. Young, and L. S. Tsimring, "Plasticity and learning in a network of coupled phase oscillators," Phys. Rev. E 65, 041906 (2002).

${ }^{36} \mathrm{~T}$. Aoki, "Self-organization of a recurrent network under ongoing synaptic plasticity," Neural Netw. 62, 11-19 (2015).

${ }^{37}$ Y. Maistrenko, B. Lysyansky, C. Hauptmann, O. Burylko, and P. A. Tass, "Multistability in the Kuramoto model with synaptic plasticity," Phys. Rev. E 75, 066207 (2007).

${ }^{38}$ O. Popovych, S. Yanchuk, and P. Tass, "Self-organized noise resistance of oscillatory neural networks with spike timing-dependent plasticity," Sci. Rep. 3, 2926 (2013).

${ }^{39}$ All variables $\kappa_{i i}$ converge to $-\sin \beta$, which leads to the same constant term $\sin (\beta) \sin (\alpha) / N$ in the right hand side of Eq. (1). The latter term can be absorbed in the corotating coordinate frame.

${ }^{40} \mathrm{~S}$. Nichols and K. Wiesenfeld, "Ubiquitous neutral stability of splay-phase states," Phys. Rev. A 45, 8430-8435 (1992).

${ }^{41}$ S. H. Strogatz and R. E. Mirollo, "Splay states in globally coupled Josephson arrays: Analytical prediction of Floquet multipliers," Phys. Rev. E 47, 220-227 (1993).

${ }^{42}$ C. U. Choe, T. Dahms, P. Hövel, and E. Schöll, "Controlling synchrony by delay coupling in networks: From in-phase to splay and cluster states," Phys. Rev. E 81, 025205(R) (2010)

${ }^{43} \mathrm{O}$. Burylko and A. Pikovsky, "Desynchronization transitions in nonlinearly coupled phase oscillators," Physica D 240, 1352-1361 (2011).

${ }^{44}$ P. Ashwin, C. Bick, and O. Burylko, "Identical phase oscillator networks: Bifurcations, symmetry and reversibility for generalized coupling," Front. Appl. Math. Stat. 2, 1-7 (2016).

${ }^{45}$ Y. Maistrenko, B. Penkovsky, and M. Rosenblum, "Solitary state at the edge of synchrony in ensembles with attractive and repulsive interactions," Phys. Rev. E 89, 3-7 (2014).

${ }^{46}$ F. Fröhlich, "Synaptic plasticity," in Network Neuroscience (Elsevier, 2016), pp. $47-58$.

${ }^{47}$ S. Boyd and L. Vandenberghe, Convex Optimization (Cambridge University Press, 2004) 